\title{
Ultraviolet and X-ray variability of active galactic nuclei with Swift
}

\author{
D. J. K. Buisson, ${ }^{1 \star ~ A . ~ M . ~ L o h f i n k, ~}{ }^{1}$ W. N. Alston ${ }^{1}$ and A. C. Fabian. ${ }^{1}$ \\ ${ }^{1}$ Institute of Astronomy, Madingley Road, Cambridge CBз OHA
}

Accepted 2016 September 27. Received 2016 September 19; in original form 2016 August 05

\begin{abstract}
We analyse a sample of 21 active galactic nuclei (AGN) using data from the Swift satellite to study the variability properties of the population in the X-ray, UV and optical band. We find that the variable part of the UV-optical emission has a spectrum consistent with a powerlaw, with an average index of $-2.21 \pm 0.13$, as would be expected from central illumination of a thin disc (index of $-7 / 3$ ). We also calculate the slope of a powerlaw from UV to X-ray variable emission, $\alpha_{\mathrm{OX}}$,Var; the average for this sample is $\alpha_{\mathrm{OX}}$.Var $=-1.06 \pm 0.04$. The anticorrelation of $\alpha_{\mathrm{OX}}$ with the UV luminosity, $L_{\mathrm{UV}}$, previously found in the average emission is also present in the variable part: $\alpha_{\mathrm{OX}}$,Var $=$ $(-0.177 \pm 0.083) \log \left(L_{\nu, \operatorname{Var}}(2500 \AA)\right)+(3.88 \pm 2.33)$. Correlated variability between the emission in X-rays and UV is detected significantly for 9 of the 21 sources. All these cases are consistent with the UV lagging the X-rays, as would be seen if the correlated UV variations were produced by the reprocessing of X-ray emission. The observed UV lags are tentatively longer than expected for a standard thin disc.
\end{abstract}

Key words: galaxies: active - galaxies: nuclei - accretion, accretion discs - black hole physics - ultraviolet:galaxies - X-rays: galaxies

\section{INTRODUCTION}

The structure of AGN is difficult to determine in part because their small size means they cannot be resolved by current instruments. Fortunately, this small size implies a short light-crossing time and hence AGN emission can vary on observable time-scales. Observations of variations in the emission from AGN have shown that they do indeed vary on all time-scales and at all wavelengths probed. The nature of these variations may be used to infer properties of the structure of AGN.

Our fundamental picture of this structure is that the central regions of AGN comprise an accretion disc principally emitting thermally in UV (Pringle 1981) and a central hot corona which Compton upscatters some of these photons to X-rays (Haardt \& Maraschi 1991). A fraction of the $\mathrm{X}$-rays are then emitted back towards the disc, which heats it, increasing its UV emission (Lightman \& White 1988). In these two ways, the X-ray and UV emission is linked and studying the details of the interaction can retrieve information about the nature of the UV and X-ray emitting regions.

The variability of the emission is strongest and occurs over the shortest time-scales at high energies (Mushotzky et al. 1993), indicating that the hard X-rays

* E-mail: djkb2@ast.cam.ac.uk are produced on the smallest scales. X-ray variability studies have become a field of their own, mapping the innermost regions around the black hole (e.g. Fabian et al. 2009; Vaughan et al. 2011; McHardy 2013; Uttley et al. 2014; Alston et al. 2014; Kara et al. 2016). UV and optical measurements over longer time-scales form the basis for studies at longer wavelengths (e.g. Cackett et al. 2007; Cameron et al. 2012), allowing a larger region of the accretion flow to be probed. The relation of the two bands is also studied (e.g. review by Gaskell \& Klimek 2003).

While variability studies do their part in enhancing our understanding of the innermost regions of AGN, studying the time-averaged emission in great detail also provides crucial information. The time-averaged UV spectra of AGN (e.g. Shull et al. 2012; Telfer et al. 2002; Vanden Berk et al. 2001; Francis et al. 1991; Schneider et al. 1991) have been measured for many different samples and wavelength ranges. When the continuum is fit with a powerlaw, $F_{\lambda} \propto \lambda^{\alpha}$, the quasars of SDSS have $\alpha=-1.56$ over $1300-5000 \AA$ (Vanden Berk et al. 2001). This is softer than the spectrum of a thin accretion disc $(\alpha=-7 / 3$, Shakura \& Sunyaev (1973)), although the variable part of the spectrum of NGC 7469 has been found to be consistent with that value (Collier et al. 1999). The difference from the theoretical value may be influenced by the strength of absorption in the UV band (e.g. Meléndez et al. 2011), the presence of 
strong emission lines (e.g. Krolik \& Kallman 1988) and host starlight (e.g. Bentz et al. 2006, 2009). Cackett et al. (2007) study optical AGN spectra and derive the reddening values necessary for the difference spectra to match a thin disc spectrum. Their reddening values match those from the flux-flux or Balmer decrement method, indicating that the variable spectrum is indeed shaped like that of a thin disc. Softer spectra are found at shorter wavelengths: $\alpha$ is $-1.32 \pm 0.14$ over $1200-1750 \AA$ and $-0.59 \pm 0.21$ over $550-1000 \AA$ (both Shull et al. 2012), suggesting the presence of a turn over at wavelengths probing the highest temperatures in the disc. The high-energy cut-off in the coolest sources may also redden the average spectral index at longer wavelengths.

When the mean UV emission is compared with that of the X-rays, the power is found to be tightly correlated: the X-ray luminosity scales as $L_{\mathrm{X}} \propto L_{\mathrm{UV}}^{k}$ with $k=0.5-0.8$ (e.g. Lusso \& Risaliti 2016; Steffen et al. 2006; Vignali et al. 2003) which results in the UV $(2500 \AA)$ to X-ray $(2 \mathrm{keV})$ slope, $\alpha_{\mathrm{OX}}$, being anticorrelated with luminosity: $\alpha_{\mathrm{OX}}=$ $a \log L_{\mathrm{UV}}+$ const, $-0.2 \lesssim a \lesssim-0.1$ (Vagnetti et al. 2010; Just et al. 2007; Strateva et al. 2005). This relation suggests that the processes producing the UV and X-ray radiation are closely related, as would be expected for an accretion disc-corona system.

The link between the X-ray and different UV bands can also be studied by comparing their correlation for a given source across time. Lags between changes in each band are interpreted as being due to the light travel time between the regions responsible for the emission in the different bands and hence the distances between them can be inferred.

Such lags have been sought in various sources (e.g. Shemmer et al. 2001; Maoz et al. 2000) and compared to the predictions for a steady state accretion disc (Shakura \& Sunyaev 1973). Where lags are found, they often disagree with thin disc theory, usually showing a longer lag than expected.

Before precision cosmology provided a largely unquestioned value of $H_{0} \simeq 70 \mathrm{~km} \mathrm{~s}^{-1} \mathrm{Mpc}^{-1}$, the luminosity of a standard disc was used to provide a distance modulus and hence $H_{0}$ (Collier et al. 1999). However, the disc sizes from the measured lags implied $H_{0}=$ $42 \pm 9 \mathrm{~km} \mathrm{~s}^{-1} \mathrm{Mpc}^{-1}$ (Collier et al. 1999) or $H_{0}=44 \pm$ $5 \mathrm{~km} \mathrm{~s}^{-1} \mathrm{Mpc}^{-1}$ (Cackett et al. 2007), so the disc is not as bright as is expected for its size. Other studies also find deviations from a standard disc. For example, studies of NGC 5548 by Edelson et al. (2015) and Fausnaugh et al. (2016) describe the disc as larger than expected for its mass and accretion rate. Similarly, Troyer et al. (2016) and Lira et al. (2015) find the best fitting accretion rate, $\dot{M}$, is unreasonably high for a standard disc model in NGC 6814 and MCG-6-30-15 respectively. The longer lags being associated with a larger disc than expected is corroborated by quasar microlensing observations, which find emitting regions a factor of a few $(2-3$, Chartas et al. (2016); $\sim 4$, Morgan et al. (2010)) larger than predicted.

However, a larger emitting region may not be the whole answer, as longer lags are not always found: McHardy et al. (2016) study the low mass AGN NGC $4395\left(3.6 \times 10^{5} M_{\odot}\right)$ and find lags which are not markedly different from standard thin disc theory.

Despite the lags often being longer than expected, the lag-wavelength relation found by these studies in the UV to
IR bands is usually consistent with the predicted $\tau \propto \lambda^{4 / 3}$ for a standard accretion disc.

Lags have also been sought in the short time-scale variability within an X-ray observation with simultaneous UV monitoring. Smith \& Vaughan (2007) analyse XMM observations of 8 sources but find no significant correlations. Arévalo et al. (2005) find the UV emission leading the Xrays by $\sim 160 \mathrm{ksec}$ (1.9 days) in a $430 \mathrm{ksec}$ observation, although this lag is a large fraction of the observation length.

These studies of lags in individual sources have shown that, at least for some sources, the reprocessing of X-ray radiation does not behave as expected for a centrally illuminated thin disc. A study of many sources has the potential to show what proportion of sources has a longer lag and whether this correlates with other AGN properties.

The Swift satellite (Gehrels et al. 2004), principally designed for the detection of GRBs, is ideal for such broadband variability analysis: it has detectors for X-rays (Burrows et al. 2005) and ultraviolet/optical emission from 1700-6000 A (Roming et al. 2005). Since it has been operating for more than a decade, many AGN lightcurves covering time-scales of several years are available.

Here, the amount of variability in the UV and X-rays and the time differences between them are analysed for a sample of AGN to determine properties of AGN as a population. Emission from the $V$-band to X-rays is included to consider a large extent of the accretion disc.

The choice of sources and observations and the reduction of data is described in Section 2. The methods and results of the analysis are described in Section 3. In particular, the UV variability is considered in Section 3.1 and the X-ray in Section 3.2. The bands are compared in terms of power in Section 3.3 and time lags are explored in Section 3.4. These results are interpreted in Section 4. Comments on individual sources are given in Section 5 .

\section{OBSERVATIONS AND DATA REDUCTION}

Our sample consists of all those AGN from the CAIXA sample (Bianchi et al. 2009) with at least 20 Swift observations by September 2015. CAIXA comprises all unobscured radioquiet AGN with public targeted XMM-Newton observations as of March 2007. The radio-loud AGN 3C 120 was also included to test whether radio-loud AGN have grossly different properties and the Seyfert 1 galaxy Zw 229-15 was included as a large number of Swift observations was present in the archive. This provides 21 sources, shown in Table 1 along with their mass and reddening.

Our analysis uses the data from both telescopes on board of Swift (Gehrels et al. 2004): the UV (UVOT, Roming et al. 2005) as well as the X-ray (XRT, Burrows et al. 2005) telescope.

The X-ray light curves used in this work were produced using the automatic pipeline of the UK Swift Science Data Centre (Evans et al. 2007, 2009). Light curves were produced with a resolution of one bin per observation in 8 fine energy bands $(0.3-0.6 \mathrm{keV}, 0.6-0.9 \mathrm{keV}, 0.9-$ $1.2 \mathrm{keV}, 1.2-1.5 \mathrm{keV}, 1.5-3.0 \mathrm{keV}, 3.0-5.0 \mathrm{keV}, 5.0-7.0 \mathrm{keV}$, $7.0-10 \mathrm{keV}$ )and the broader bands $0.3-10 \mathrm{keV}, 0.3-1.5 \mathrm{keV}$ and $1.5-10 \mathrm{keV}$. For our analysis we used the default grade selection. When converting counts to flux, we correct for 
Table 1. List of sources in our sample, with number of observations, black hole mass and reddening values for each object

\begin{tabular}{|c|c|c|c|c|c|c|c|}
\hline Source & $\begin{array}{c}\text { Number of } \\
\text { Observations }\end{array}$ & $\begin{array}{c}\text { UV bands with } \\
\geq 10 \text { Observations }\end{array}$ & $\log _{10}\left(M_{\mathrm{BH}}\right)$ & Ref. & $\begin{array}{l}E(B-V) \\
(\text { Galactic) }\end{array}$ & $\begin{array}{l}E(B-V) \\
\text { (Intrinsic) }\end{array}$ & Ref. \\
\hline NGC 5548 & 744 & 6 & $7.59_{-0.21}^{+0.24}$ & $\mathrm{P}$ & 0.0171 & 0.152 & $\mathrm{G}$ \\
\hline MRK 335 & 339 & 6 & $7.23_{-0.04}^{+0.04}$ & $\mathrm{~B}$ & 0.0307 & 0.00 & $\mathrm{C}$ \\
\hline NGC 7469 & 224 & 6 & $6.96_{-0.05}^{+0.05}$ & $\mathrm{~B}$ & 0.0591 & 0.09 & $\mathrm{C}$ \\
\hline FAIRALL 9 & 168 & 6 & $8.41_{-0.09}^{+0.11}$ & $\mathrm{P}$ & 0.0223 & 0 & $\mathrm{~N}$ \\
\hline 1H 0707-495 & 119 & 1 & $6.72_{-0.5}^{+0.5}$ & $\mathrm{~Pa}$ & 0.0816 & 0 & $\mathrm{~N}$ \\
\hline MCG-6-30-15 & 105 & 1 & $6.7_{-0.2}^{+0.1}$ & $\mathrm{P}$ & 0.0521 & 0.54 & Wa \\
\hline MRK 766 & 100 & 6 & $6.82_{-0.06}^{+0.05}$ & $\mathrm{P}$ & 0.0169 & 0.613 & $\mathrm{G}$ \\
\hline ARK 120 & 90 & 2 & $8.18_{-0.06}^{+0.05}$ & $\mathrm{P}$ & 0.1094 & 0.04 & $\mathrm{C}$ \\
\hline IRAS $13224-3809$ & 70 & 1 & $6.76_{-0.5}^{+0.5}$ & $\mathrm{Z}$ & 0.0601 & 0.628 & $\mathrm{Po}$ \\
\hline PG $1211+143$ & 68 & 6 & $8.17_{-0.15}^{+0.11}$ & $\mathrm{P}$ & 0.0293 & 0 & $\mathrm{G}$ \\
\hline NGC 4051 & 59 & 1 & $6.13_{-0.16}^{+0.12}$ & $\mathrm{P}$ & 0.011 & 0.12 & Wi \\
\hline NGC 3516 & 58 & 1 & $7.40_{-0.06}^{+0.04}$ & B & 0.0359 & 0.15 & $\mathrm{C}$ \\
\hline MRK 1383 & 35 & 2 & $9.01_{-0.07}^{+0.11}$ & B & 0.0275 & 0 & $\mathrm{~N}$ \\
\hline PG $1247+267$ & 33 & 1 & $8.91_{-0.17}^{+0.15}$ & $\mathrm{~T}$ & 0.0112 & 0 & $\mathrm{~N}$ \\
\hline Н $0557-385$ & 28 & 1 & $7_{-1}^{+1}$ & $\mathrm{~A}$ & 0.0375 & 0.511 & $\mathrm{Ki}$ \\
\hline MRK 509 & 27 & 3 & $8.05_{-0.04}^{+0.04}$ & B & 0.0493 & 0.11 & $\mathrm{C}$ \\
\hline MRK 841 & 26 & 6 & $7.88_{-0.1}^{+0.04}$ & $\mathrm{~V}$ & 0.0255 & 0 & $\mathrm{Wi}$ \\
\hline PDS 456 & 22 & 2 & $8.91_{-0.5}^{+0.5}$ & $\mathrm{Z}$ & 0.4450 & 0 & $\mathrm{~N}$ \\
\hline IC $4329 A$ & 20 & 6 & $8.34_{-0.3}^{+0.5}$ & M & 0.0501 & 0.98 & M \\
\hline $3 \mathrm{C} 120$ & 71 & 5 & $7.75_{-0.04}^{+0.04}$ & B & 0.2558 & 0.05 & $\mathrm{H}$ \\
\hline Zw 229-15 & 71 & 1 & $6.91_{-0.12}^{+0.08}$ & B & 0.0615 & 0 & $\mathrm{~N}$ \\
\hline
\end{tabular}

A: Ashton et al. (2006), B: Bentz \& Katz (2015), M: Markowitz (2009), P: Peterson et al. (2004), Pa: Pan et al. (2016), T: Trevese et al. (2014), V: Vestergaard (2002), Z: Zhou \& Wang (2005).

C: Cackett et al. (2007), G: Grupe et al. (2010), H: Hjorth et al. (1995), Ki: Kishimoto et al. (2011), M: Marziani et al. (1992), N: No value found in literature $-E(B-V)=0$ assumed, Po: Polletta \& Courvoisier (1999), Wi: Winter et al. (2010), Wa: Ward et al. (1987)

Galactic absorption using values from the LAB survey (Kalberla et al. 2005).

The UVOT observations were taken in different filters ( $V: 5440 \AA$ А $B: 4390 \AA, U: 3450 \AA, W 1: 2510 \AA, M 2: 2170 \AA$, W2: $1880 \AA$ ) and we reduce each filter individually for each observation. We started our UVOT reduction from the level II image files, performing photometry with the tool uvotsource. To obtain the source counts, we assumed a circular source region with a 5 arcsec radius and a, also circular, background region with a 15 arcsec radius. Some areas of the UVOT detector have shown spuriously low fluxes (Edelson et al. 2015), so we excluded observations where the source region overlaps these bad areas. Count rates were converted to fluxes for each source using calibration factors from Poole et al. (2008).

Where necessary, the UV emission for each source was dereddened using the idl tools ccmunred (Cardelli et al. 1989; O'Donnell 1994) for Galactic dust and mszdst ${ }^{1}$ for host absorption, with the extinction law from the LMC (Pei 1992).

UV/optical fluxes include a significant contribution from the host galaxy. Removing this component is difficult and requires high resolution images of the host galaxy. Here, we avoid the problem by using methods which are not affected by the additional constant host flux.

The luminosity distance was calculated with $H_{0}=$ $70 \mathrm{kms}^{-1} \mathrm{Mpc}^{-1}$. The relative luminosities are not sensi-

1 https://heasarc.gsfc.nasa.gov/xanadu/xspec/models/dust.html tive to cosmological parameters since all sources apart from PG1247+267 $(z=2.038)$ are at low redshift $(z \ll 1)$.

\section{RESULTS}

\subsection{UV variability}

We initially consider the variability of the 6 Swift $\mathrm{UV} /$ optical bands.

\subsubsection{Presence of variability}

To characterise the variability of the luminosity in each band, the measured standard deviation $(\sigma)$ and mean square error $\left(\overline{\epsilon^{2}}\right)$ were calculated and the intrinsic variability was estimated as the error-corrected rms variability, $L_{\text {Var }}=$ $\sqrt{\sigma^{2}-\epsilon^{2}}$ (Nandra et al. 1997; Edelson et al. 2002). We only consider UV bands with at least 10 data points to remove lightcurves where the uncertainty would be excessively dominated by the stochastic variations due to sampling.

$\mathrm{X}$-ray studies usually consider the fractional (rms) variablity, $F_{\text {Var }}=L_{\text {Var }} / \bar{L}$ (although this is usually calculated directly from the measured count rates), where $\bar{L}$ is the mean of the measured lightcurve, to avoid the effects of absorption. Absorption is a multiplicative effect which changes $L_{\text {Var }}$ and $\bar{L}$ by the same factor, so their ratio is unchanged. In the UV, host galaxy contamination is a more significant problem, so $\bar{L}$ from the AGN alone is not precisely known. This constant addition to the measured $\bar{L}$ has no effect on the variance of 


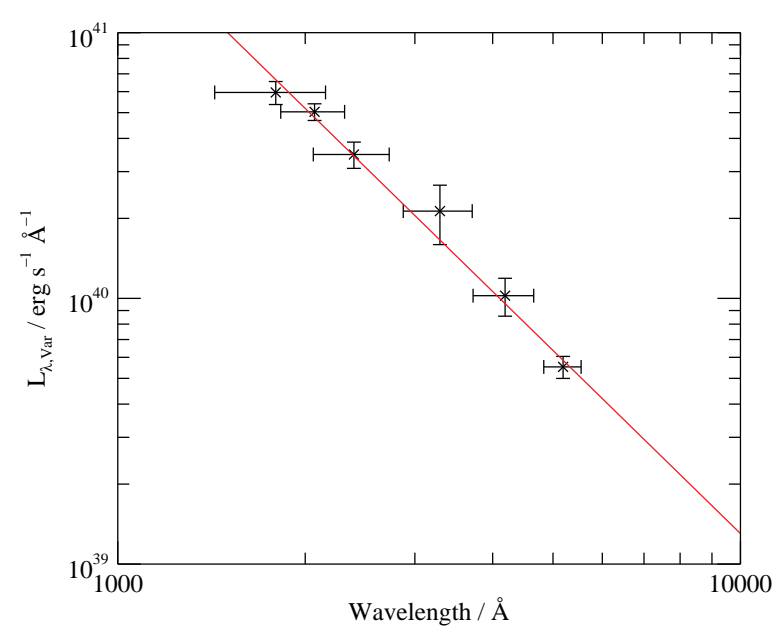

Figure 1. The spectrum of the variable UV emission of Fairall 9, along with the best-fitting powerlaw, $\alpha=-2.29 \pm 0.10$.

the lightcurve. Hence, we prefer the absolute luminosity variance. The standard deviation (rms variability) is preferred to the variance because of its more familiar dimension.

We define a source as variable if the variations in measured flux are larger than would be expected due to measurement errors alone. The approximate error in the lightcurve variability is given by equation (1) (Vaughan et al. 2003, eq. 11 ), where $N$ is the number of datapoints.

$\operatorname{err}\left(L_{\mathrm{Var}}^{2}\right)=\frac{1}{\sqrt{N}} \sqrt{\left(\sqrt{2} \bar{\epsilon}^{2}\right)^{2}+\left(2 \sqrt{\overline{\epsilon^{2}}} L_{\mathrm{Var}}\right)^{2}}$

We assess the variability for each source and band separately. In four bands out of 90 with sufficient data $(\geq 10$ points) to calculate variability (IC 4329A B, W2; MRK 766 M2; PDS $456 \mathrm{W1}$ ), the error was greater than the measured variability. Since the measured variability is subject to random variations depending on when a source is observed, a varying source may by chance be observed when it varies little. Since there is no reason to expect this small fraction of bands to not be varying, these bands were not excluded from further analysis to avoid biasing results towards observations which happened to catch higher variability.

\subsubsection{UV variability spectra}

For 12 sources, $L_{\lambda, \text { Var }}$ measurements exist in at least 2 wavebands; for these, we produced a spectrum of the variable component of the emission. The variable UV spectrum of Fairall 9, which is typical of the sample, is shown in Fig. 1, fitted with a powerlaw. Spectra of the whole sample are shown in Appendix B.

To characterise the shape of the spectrum, the spectrum of variable luminosity was fitted with a powerlaw, $L_{\lambda, \operatorname{Var}} \propto \lambda^{\alpha}$. We performed a chi-square minimisation with mpfit in IDL in the logarithmic domain. The wavelengths of each band were taken as the nominal central value. Errors of the luminosity variability were estimated from equation (1). Values for $\alpha$ are given in Table 2 .

In most sources, the powerlaw provides a good fit; however, for 3C 120, Mrk 766 and PG $1211+143$ the reduced $\chi^{2}$
Table 2. Index of powerlaw fit to variable part of UV spectrum, with $1 \sigma$ errors. Most sources are consistent with $-2>\alpha>-2.33$, as predicted for a thin accretion disc

\begin{tabular}{lccc}
\hline Source & Index, $\alpha$ & $\chi_{\text {red }}^{2}$ & d.o.f. \\
\hline 3C 120 & $-2.21 \pm 0.10$ & 5.10 & 3 \\
ARK 120 & $-2.41 \pm 0.83$ & - & 0 \\
Fairall 9 & $-2.29 \pm 0.10$ & 0.94 & 4 \\
IC 4329A & $-3.14 \pm 0.27$ & 1.78 & 1 \\
MRK 335 & $-2.27 \pm 0.11$ & 2.14 & 4 \\
MRK 509 & $-1.96 \pm 2.41$ & 0.04 & 1 \\
MRK 766 & $-3.57 \pm 0.09$ & 34.31 & 4 \\
MRK 841 & $-2.11 \pm 0.16$ & 0.59 & 4 \\
MRK 1383 & $-2.81 \pm 0.88$ & - & 0 \\
NGC 5548 & $-2.71 \pm 0.07$ & 0.67 & 4 \\
NGC 7469 & $-3.13 \pm 0.39$ & 1.65 & 3 \\
PG 1211+143 & $-2.01 \pm 0.15$ & 9.90 & 4 \\
\hline
\end{tabular}

value is unacceptably high. Exploring the reasons for this, we find that in Mrk 766, the W2 measurement is much higher than would be expected from extrapolating a powerlaw fitted to the remaining values. Since for Mrk 766 the W2 band has a large number of samples from 2012-13 which are not taken in the other filters, the W2 variability measurement may be increased by the difference in variability in the different epochs. In PG1211+143, the $U$-band is somewhat higher than expected and the M2 much lower. In 3C 120, the $U$ and M2-bands are both higher than the fit. Since these sources show scatter rather than curvature, it is possible that the errors have been underestimated.

From the fits, we find an average slope of $\alpha=-2.6 \pm 0.8$. For all sources apart from IC 4329A, MRK 766, NGC 5548 and NGC 7469, the index is consistent with $\alpha=-2$ to -2.33 , as predicted by Davis et al. (2007) for a thin accretion disc. The variable spectrum of NGC 5548 has also been measured by Edelson et al. (2015) with a subset of the data used here, finding $\alpha=-1.98 \pm 0.20$ assuming no intrinsic reddening (this is consistent with our value before dereddening). For IC 4329A and Mrk 766, the intrinsic reddening is strong (Table 1), so the uncertainty in the reddening may allow their indices to be consistent with $\alpha=-2$ to -2.33 . Collier et al. (1999) also measured the variable spectrum of NGC 7469, finding an index consistent with $\alpha=-2.33$.

For the sources with $E(B-V) \leq 0.05$ and excluding those sources with very large uncertainties $(\Delta \alpha>0.75)$, the average index is $\alpha=-2.21 \pm 0.13$. We consider this our best estimate of the slope.

In order to investigate the nature of the variability on different time-scales and ensure that the non-uniformly sampled lightcurves do not bias the results based on the different time-scales sampled in different observations, the lightcurves were also split into sections of different lengths. The index of the variable spectrum was calculated in the same way as for the full lightcurve. For each section length, we calculate the average index of all sections. For Fairall 9, which has enough data to split on many time-scales, Fig. 2 shows the spectral index as a function of section length. The index converges towards the value calculated from the full lightcurve as the section length increases, which justifies the use of the full lightcurve.

Finally, we consider how the spectra of variable power 


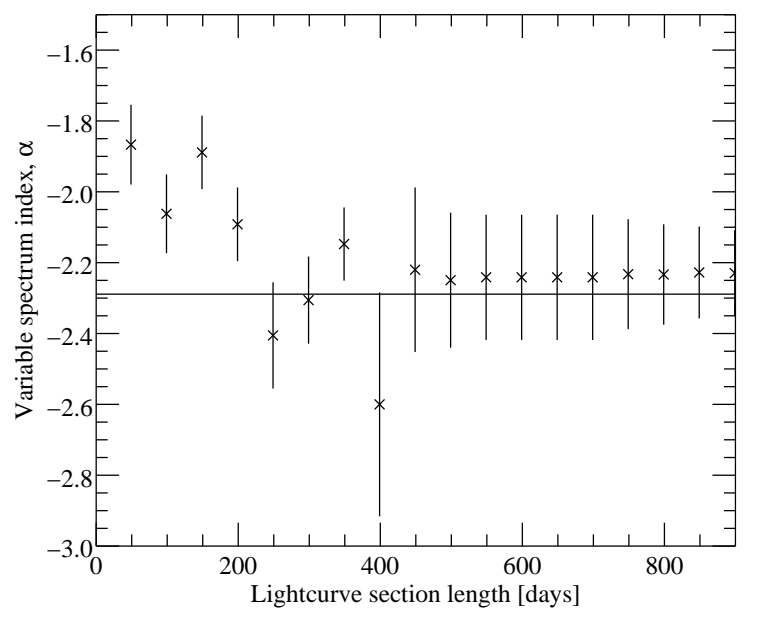

Figure 2. Plot of index of variable spectrum of Fairall 9 against duration of lightcurve used to calculate variability. The index shown at each timescale is the average over all sections of given length. The horizontal line shows the value for the full lightcurve. The index appears to converge to the value measured from the full lightcurve as section length increases.

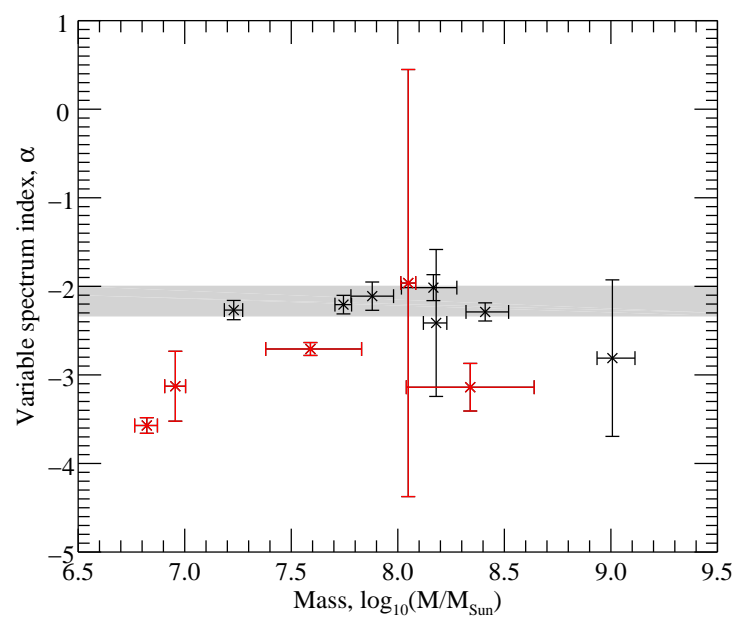

Figure 3. Plot of spectral index of variable emission against black hole mass. Most sources are consistent with an index of $-2>\alpha>$ -2.33 (indicated by the shaded region), as predicted for a thin accretion disc. Those sources with a steeper spectrum tend to have been the most dereddened: sources with $E(B-V)_{\text {int }}>0.05$ are shown in red. No correlation with mass is apparent $(r=0.36)$.

depend on black hole mass. Plotting the spectral indices, $\alpha$, of the variable part of the spectrum against mass (Fig. 3) shows no clear correlation $(r=0.36)$. Fig. 4 shows the variable luminosity in each UV band against black hole mass, $M_{\mathrm{BH}}$. Sources with observations in all 6 bands are shown in black and the remaining sources in grey. It is apparent that $L_{\text {Var }}$ increases with mass. While the black points show a tight correlation, this is a selection effect: the power is also dependent on the Eddington ratio and, for this subsample, the Eddington ratio smoothly decreases with mass (Table 4). This flattens the observed correlation so the slope of the relationship is not meaningful. The effect of scattered Eddington ratios can be seen in the greater scatter when including the remaining sources (grey points). The correlations between $M_{\mathrm{BH}}$ and $L_{\mathrm{Var}}$ seen in the black points do show that the scaling with mass is consistent between the different bands, as would be expected if the spectral shape is constant.

\subsection{X-ray Variability}

To compare the variability of the thermal disc emission with that of the coronal emission, we also calculated the excess variance in the $8 \mathrm{X}$-ray bands. We estimated the error in the excess variance due to measurement error from simulated lightcurves. To do this, we simulated lightcurves by adding values from a Gaussian with the same width as the error on each observation to the measured lightcurve and used the distribution of excess variance from 20000 realisations to estimate $1 \sigma$ and $2 \sigma$ confidence intervals. As the error is comparable to the measured value in some sources, we converted the confidence limits from variance to rms directly, as in Poutanen et al. (2008), rather than using differential error propagation.

The variable X-ray luminosity spectra this produces are shown alongside the UV variable spectra in Appendix B. The $\mathrm{X}$-ray variable spectra look superficially similar in shape to the mean spectra of their respective sources.

To quantify the differences between the mean and variable spectra, we fitted powerlaws to the hard $(1.5-10 \mathrm{keV})$ $\mathrm{X}$-ray band for each source. For consistency with other $\mathrm{X}$-ray measurements, we use the photon index defined as $N(E) \propto E^{-\Gamma}$ (note that this converts from the wavelength spectral index as $\Gamma=\alpha_{\mathrm{X}}+3$ ). These photon indices, $\Gamma_{V a r}$, are compared to the indices of the mean spectra over the same band, $\Gamma_{\mathrm{Avg}}$, in Fig. 5 . This shows that the two indices are well correlated $(r=0.81)$ and the variable spectra are softer than the average spectra over the $1.5-10 \mathrm{keV}$ band. We estimate the relation between the variable and average indices with a linear function; the best fitting is $\Gamma_{\text {Var }}=(0.97 \pm 0.07) \Gamma_{\text {Avg }}+(0.36 \pm 0.08)$, compatible with a constant offset. Fitting for a constant offset gives $\Delta \Gamma=0.28 \pm 0.02$ between average and variable index.

\subsection{Comparison of UV and X-ray variable power}

To help understand the interactions between the disc and corona, we compared the power of the variability in the Xray $\left(L_{\mathrm{X}, \mathrm{Var}}\right)$ and $\mathrm{UV}\left(L_{\mathrm{UV}, \mathrm{Var}}\right)$.

We first considered the variable power in the directly measured energy ranges, $0.3-10 \mathrm{keV}$ (X-ray) and $1500-$ $5815 \AA$ (UV). The X-ray variability power was calculated from a direct sum over the power in each energy bin. Since the UV bands do not fully and evenly cover their overall wavelength range, we used a powerlaw fit to the datapoints integrated over the total range of the Swift filters. The variable power in the X-ray and UV is plotted in Fig. 6; this shows that the UV and X-ray power is broadly comparable. There is a strong correlation $(r=0.74)$ between the two quantities with the UV power increasing somewhat faster than the X-ray power. Approximating the relation with a powerlaw $L_{\mathrm{X}, \operatorname{Var}} \propto L_{\mathrm{UV}, \operatorname{Var}}^{\beta}$ gives a best fit of $\beta=0.66 \pm 0.22$. 


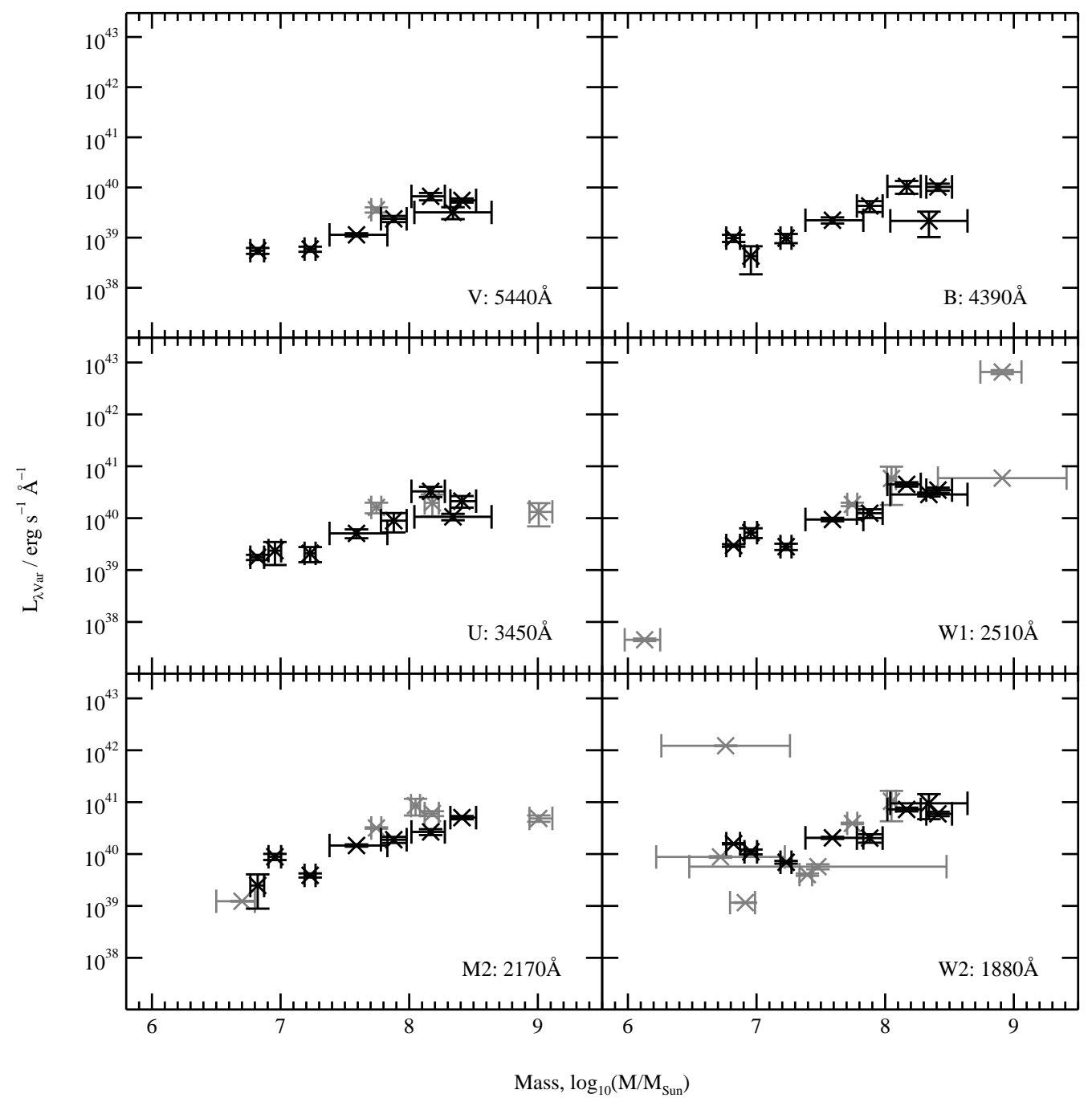

Figure 4. Plot of variable luminosity, $L_{\mathrm{Var}}$, against black hole mass in each waveband. Sources in black are observed in all bands, other sources are in grey.

This is consistent with the relationship between the average luminosities, for which $\beta=0.72 \pm 0.01$ or $0.75 \pm 0.06$ have been found by Steffen et al. (2006) and Vignali et al. (2003) respectively.

We also consider the relative specific luminosity, using the definition of $\alpha_{\mathrm{OX}}$ applied to the variable part of the emission. $\alpha_{\mathrm{OX}}$ is the index of a powerlaw between the specific luminosity at $2500 \AA$ and $2 \mathrm{keV}$ (e.g. Vagnetti et al. 2010):

$$
\begin{aligned}
\alpha_{\mathrm{OX}} & =\frac{\log \left(L_{\nu}(2 \mathrm{keV}) / L_{\nu}(2500 \AA)\right)}{\log \left(\nu_{2} \mathrm{keV} / \nu_{2500 \AA}\right)} \\
& =0.3838 \log \left(\frac{L_{\nu}(2 \mathrm{keV})}{L_{\nu}(2500 \AA)}\right)
\end{aligned}
$$

Here, we measure $\alpha_{\mathrm{OX}}$, Var from the variable specific luminosities. The average is $\alpha_{\mathrm{OX}, \mathrm{Var}}=-1.06 \pm 0.04$, which is flatter than some measurements of $\alpha_{\mathrm{OX}}$ measured from mean spectra of similarly bright sources (e.g. $-1.32 \pm 0.03$, Steffen et al. 2006) but similar to values found in $\mathrm{Xu}$ (2011), which includes some sources which are in our sample. $\alpha_{\text {OX }}$, var for each source is shown against specific variable UV luminosity at $2500 \AA$ in Fig. 7. These are anticorrelated, $r=-0.73$, and the least-squares fit is

$\alpha_{\mathrm{OX}, \operatorname{Var}}=(-0.177 \pm 0.083) \log L_{\nu, \operatorname{Var}}(2500 \AA)+(3.88 \pm 2.33)$

This relation has a slope within those found by previous authors for the mean spectrum, such as that by Gibson et al. (2008):

$\alpha_{\mathrm{OX}}=(-0.217 \pm 0.036) \log L_{2500 \AA}+(5.075 \pm 1.118)$

or the flatter relation found by Just et al. (2007):

$\alpha_{\mathrm{OX}}=(-0.140 \pm 0.007) \log L_{2500 \AA}+(2.705 \pm 0.212)$

$\mathrm{Xu}$ (2011) studied low-luminosity AGN and found:

$\alpha_{\mathrm{OX}}=(-0.134 \pm 0.031) \log L_{2500 \AA}+(2.406 \pm 0.785)$

Grupe et al. (2010) also analysed a sample of local $(z<$ $0.35)$ sources with Swift, so provide the most similar reference sample:

$\alpha_{\mathrm{OX}}=(-0.114 \pm 0.014) \log L_{2500 \AA}+(1.177 \pm 0.305)$ 


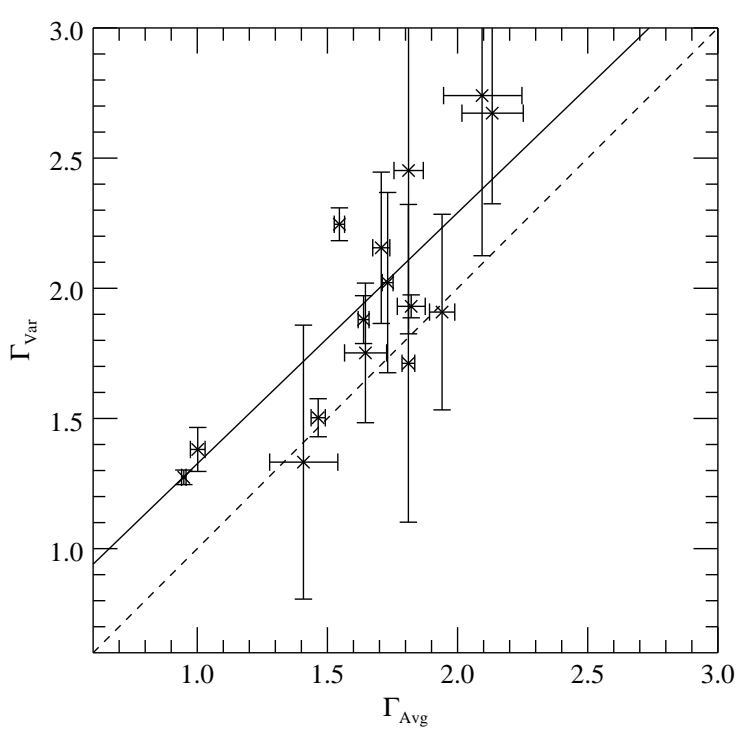

Figure 5. Photon index $(\Gamma)$ of powerlaw fit to average and variable parts of $1.5-10 \mathrm{keV}$ X-ray spectra. Solid line: best fit; dashed line: equal variable and average indices. The variable part is usually softer than the average spectrum, with an average $\Delta \Gamma=0.28 \pm 0.02$ ( $1 \sigma$ error $)$.

However, Vagnetti et al. (2013) corrected for the galactic dilution in this sample and found:

$\alpha_{\mathrm{OX}}=(-0.135 \pm 0.015) \log L_{2500 \AA}+(2.645 \pm 0.446)$

While our value is within the range found by previous authors for the mean spectrum, the spread in indices from measurements of mean spectra is much larger than the error for a given sample and therefore a reliable comparison between the $\alpha_{\mathrm{OX}}-\log L_{2500 \AA}$ relations for the mean and variable parts of the spectra would require that both be calculated from the same sample and dataset.

\subsection{Interband Lags}

So far, we have only considered the amount of variability in each band. To investigate how the variability in different bands is related in time, we calculated the correlations between the different bands.

To look for correlated variability between the X-rays (0.3-10 keV) and each of the UV bands for each source, we used the discrete cross-correlation function (DCF) (Edelson \& Krolik 1988). The X-rays were chosen as the reference band since this is the only band which is measured for every source. Since the number and frequency of observations differs widely between sources, we grouped the DCF into lag bins by number of observation pairs rather than a fixed lag width. We chose 100 points as the minimum compatible with little apparent noise in the well-sampled sources. Before calculating the DCF, a 30 day half-width boxcar running mean was subtracted from the lightcurves to remove the effects of long-term variations and highlight the expected lags of a few days (Welsh 1999).

To determine the significance of any correlations, we

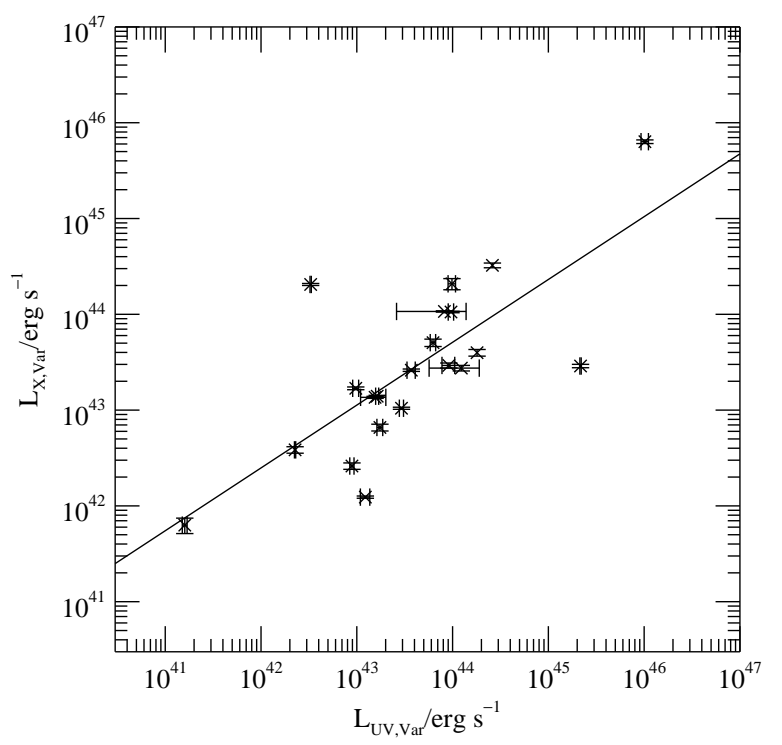

Figure 6. Plot of total UV against X-Ray variable power in measured bands: UV: $1500-5815 \AA$; X-ray: $0.3-10 \mathrm{keV}$. The line shows the best-fitting powerlaw, $L_{\mathrm{X}, \mathrm{Var}} \propto L_{\mathrm{UV}, \operatorname{Var}}^{0.66 \pm 0.22}$

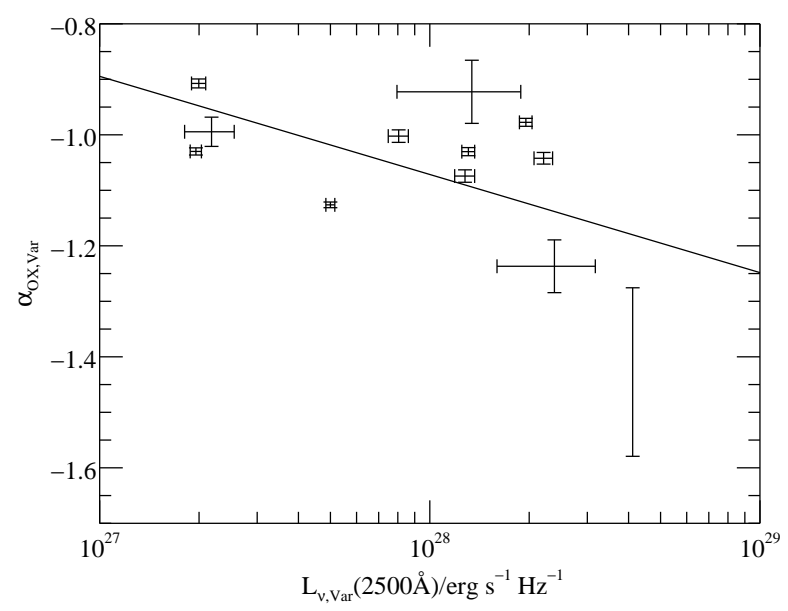

Figure 7. Plot of $\alpha_{\mathrm{OX}}, \operatorname{Var}$ against $L_{\nu, \operatorname{Var}}(2500 \AA)$. The solid line shows the least squares fit, $\alpha_{\text {OX, Var }}=(-0.177 \pm$ $0.083) \log L_{\nu, \operatorname{Var}}(2500 \AA)+(3.88 \pm 2.33)$.

used the distribution of the DCFs from 10000 uncorrelated simulated lightcurves in each band. Using the method of Timmer \& Koenig (1995), we produced lightcurves with the same power spectra as the real lightcurves and a resolution equal to the average observation length. We used X-ray power spectra from González-Martín \& Vaughan (2012) and estimated UV power spectra by fitting a powerlaw to the periodogram of the Swift data. We added the rms-flux relation by taking the exponential of the lightcurves (Uttley et al. 2005). From these regular lightcurves, we extracted count measurements at points corresponding to the actual observation times and simulated observational noise by drawing 
the final simulated data from a Poisson distribution with mean equal to these count measurements. We calculated the DCF for each UV/X-ray pair and used the distribution of the DCFs at each lag value to estimate the $95 \%$ and $99 \%$ confidence intervals outside which a correlation is unlikely to be produced by random noise. This found significant $(>99 \%)$ correlations in 9 sources out of the sample of 21. These sources either have more datapoints or a clear peak in the lightcurve, so the non-detection in the remaining sources is likely due to a lack of data quality rather than less intrinsic correlation. MCG-6-30-15 notably has many datapoints but no significant lag detection; see Section 5.3 for a detailed discussion.

Where a significant correlation was found, we estimated the potential lag between bands using the centroid of the DCF peak. To generate enough points to produce a smooth distribution to centroid, the X-ray lightcurve was linearly interpolated onto a finer grid $(\Delta t=0.1$ days $)$ and the ICCF of each UV band was measured against it (Gaskell \& Sparke 1986; Gaskell \& Peterson 1987). We used the centroid of the region with a correlation coefficient of at least 0.8 times the maximum value (e.g. Troyer et al. 2016).

Errors on the lag values obtained were estimated using subset selection/flux randomisation (Peterson et al. 1998, 2004). From 2000 realisations, we estimated $1 \sigma$ errors by percentile.

Lag values are shown in Table 3. In all 9 sources, the UV bands are consistent with a lag behind the X-rays. For the two best measured sources, NGC 5548 and Fairall 9, the lower energy bands have a longer delay. For the remaining sources, the lags are not sufficiently well constrained to determine differences in lag between the different UV bands.

We compared these lags with the theoretical lags for a thin accretion disc around a black hole of the appropriate mass and accretion rate (Shakura \& Sunyaev 1973). To do so, we calculated the accretion rate in terms of the Eddington ratio, $\dot{m}$, derived from the average hard $(2-10 \mathrm{keV}) \mathrm{X}$-ray luminosity during the Swift monitoring. We used correction factors, $\kappa$, to convert from X-ray to bolometric luminosity based on the most recent measurement in Vasudevan et al. (2010); Vasudevan \& Fabian (2009, 2007). Where a source does not have a value for $\kappa$ in any of these papers, we estimated $\dot{m}$ from the average X-ray spectral index, $\Gamma_{\text {Avg }}$, using the relation from Shemmer et al. (2008). The resulting Eddington ratios are given in Table 4 .

For each source, the lags and theoretical predictions (black lines) are plotted in Appendix D. The red lines indicate the $1 \sigma$ uncertainties due to the mass and luminosity uncertainties but do not include uncertainties in $\kappa$.

The two best-measured sources, NGC 5548 and Fairall 9, both have lags slightly longer than the predicted values but each individual value is still consistent. The remaining sources do not have enough points of sufficient quality to determine inconsistencies as individuals.

To explore any potential global deviation from the lags expected for a thin accretion disc, the lags for each source were scaled to represent the accretion disc around a black hole with a common mass, $10^{8} M_{\odot}$, and Eddington fraction, $\dot{m}=0.1$. The lags were scaled by $\tau \sim R / c \sim \dot{m}^{1 / 3} M^{2 / 3}$ (Shakura \& Sunyaev 1973).

These scaled lags are plotted in Fig. 8, along with the expected lags for a thin accretion disc (red) and the best
Table 3. Lags of each UV band behind X-Rays, with $1 \sigma$ errors.

\begin{tabular}{|c|c|c|}
\hline Source & Band & Lag/days \\
\hline 3C 120 & $\begin{array}{c}\mathrm{U} \\
\mathrm{W} 2\end{array}$ & $\begin{array}{c}0.4 \pm 4.5 \\
-0.2 \pm 2.3\end{array}$ \\
\hline ARK 120 & $\mathrm{U}$ & $2.4 \pm 2.3$ \\
\hline Fairall 9 & $\begin{array}{c}\mathrm{V} \\
\mathrm{B} \\
\mathrm{U} \\
\mathrm{W} 1 \\
\mathrm{M} 2 \\
\mathrm{~W} 2\end{array}$ & $\begin{array}{l}4.2 \pm 2.8 \\
2.7 \pm 1.9 \\
3.1 \pm 1.5 \\
2.2 \pm 1.0 \\
1.7 \pm 1.0 \\
1.7 \pm 0.8\end{array}$ \\
\hline IRAS 13224-3809 & W2 & $6.4 \pm 3.7$ \\
\hline MRK 335 & W2 & $0.0 \pm 2.9$ \\
\hline MRK 1383 & M2 & $4.3 \pm 8.5$ \\
\hline NGC 3516 & W2 & $1.6 \pm 1.5$ \\
\hline NGC 5548 & $\begin{array}{c}\mathrm{V} \\
\mathrm{B} \\
\mathrm{U} \\
\mathrm{W} 1 \\
\mathrm{M} 2 \\
\mathrm{~W} 2\end{array}$ & $\begin{array}{l}2.0 \pm 1.1 \\
1.5 \pm 0.8 \\
1.4 \pm 0.7 \\
1.0 \pm 0.7 \\
0.8 \pm 0.7 \\
0.7 \pm 0.5\end{array}$ \\
\hline NGC 7469 & $\begin{array}{c}\mathrm{U} \\
\mathrm{W} 1 \\
\mathrm{M} 2 \\
\mathrm{~W} 2\end{array}$ & $\begin{array}{c}1.1 \pm 1.0 \\
-0.3 \pm 1.2 \\
1.3 \pm 1.7 \\
0.8 \pm 0.7\end{array}$ \\
\hline
\end{tabular}

Table 4. X-ray luminosities and Eddington ratios for each source in the sample. $\dot{m}$ is calculated by converting $\log _{10} L_{2-10 \mathrm{keV}}$ to a bolometric luminosity where $\kappa$ is available. For the remaining sources, $\dot{m}$ is estimated from $\Gamma_{\text {Avg }}$.

\begin{tabular}{lccc}
\hline Source & $\log _{10} L_{2-10 \mathrm{keV} / \mathrm{erg} \mathrm{s}^{-1}}$ & $\kappa$ & $\dot{m}$ \\
\hline 1H 0707-495 & $41.90 \pm 0.01$ & & 0.37 \\
3C 120 & $44.09 \pm 0.01$ & 8.29 & 0.14 \\
ARK 120 & $43.78 \pm 0.02$ & 25.0 & 0.08 \\
Fairall 9 & $43.98 \pm 0.01$ & 10.5 & 0.03 \\
H 0557-385 & $42.76 \pm 0.02$ & & 0.02 \\
IC 4329A & $43.83 \pm 0.01$ & 14.8 & 0.04 \\
IRAS 13224-3809 & $42.34 \pm 0.06$ & & 0.68 \\
IRAS 13349+2438 & $43.64 \pm 0.05$ & & 0.07 \\
MCG-6-30-15 & $42.65 \pm 0.01$ & 22.2 & 0.16 \\
MRK 335 & $42.79 \pm 0.01$ & 102 & 0.30 \\
MRK 509 & $43.97 \pm 0.01$ & 12.5 & 0.08 \\
MRK 766 & $42.66 \pm 0.01$ & 70.5 & 0.39 \\
MRK 841 & $43.48 \pm 0.01$ & 27.4 & 0.09 \\
MRK 1383 & $44.07 \pm 0.01$ & 33.5 & 0.03 \\
NGC 3516 & $42.58 \pm 0.01$ & 17.7 & 0.02 \\
NGC 4051 & $41.03 \pm 0.01$ & 16.5 & 0.01 \\
NGC 5548 & $43.25 \pm 0.00$ & 18.8 & 0.07 \\
NGC 7469 & $43.06 \pm 0.01$ & 38.7 & 0.39 \\
PDS 456 & $44.56 \pm 0.01$ & & 0.24 \\
PG 1211+143 & $43.55 \pm 0.02$ & 92 & 0.18 \\
PG 1247+267 & $45.82 \pm 0.04$ & & 0.22 \\
Zw 229-15 & $42.56 \pm 0.02$ & & 0.12 \\
\hline
\end{tabular}




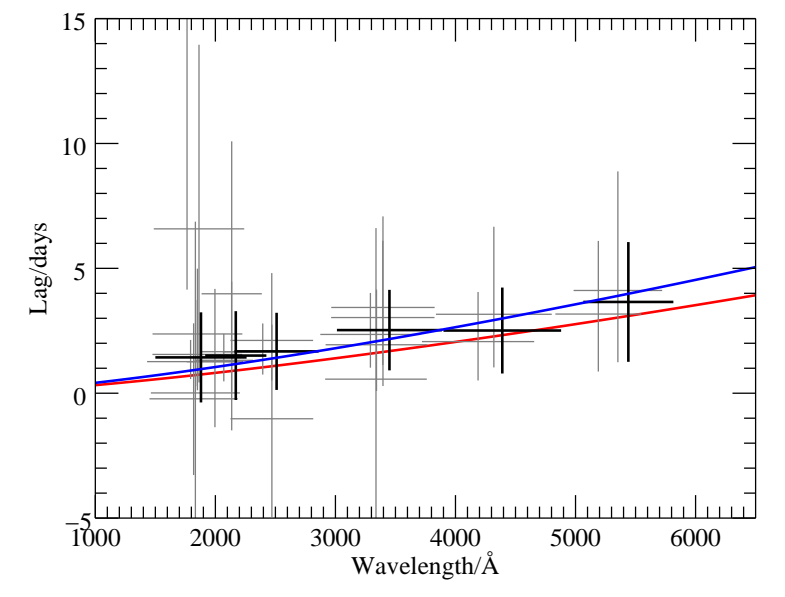

Figure 8. Lags relative to X-rays for each source scaled to a black hole mass of $10^{8} M_{\odot}$ and Eddington rate $\dot{m}=0.1$. The red (lower) line shows the lags expected for a thin disc; the blue (upper) line shows the best fitting scaled lags.

fitting size for the measured lags (blue). In each band, the average lag (corrected to the rest wavelength of the band) is shown in black. For every band, the measured lag is greater than the theoretical by an average factor of 1.3. However, the overall deviation is only $1.5 \sigma$.

\section{DISCUSSION}

\subsection{Summary of results}

From our study of 21 AGN monitored by Swift over timescales of several years in bands from optical through to Xray, we find that:

- The UV variable spectra are consistent with a powerlaw. The average index is $\alpha=-2.21 \pm 0.13$.

- The amount of variable power increases with mass at the same rate for all UV bands.

- The UV variable luminosity and the index from UV to X-rays, $\alpha_{\mathrm{OX}}$, are anticorrelated: $\alpha_{\mathrm{OX}}=(-0.177 \pm 0.083) \log L_{\mathrm{UV}, \mathrm{Var}}+(3.88 \pm 2.33)$

- The variable X-ray spectra are softer than the average spectra of their respective sources by $\Delta \Gamma=0.28 \pm 0.06$.

- Significant correlations between X-ray and UV variability are detectable in 9 sources. The remaining sources are generally less well sampled and any correlated variability is too weak to detect with the current data.

- Every lag measurement is consistent with variations in the UV lagging behind the X-rays.

\subsection{Comparison with average spectra}

In the unified AGN model (Antonucci 1993; Urry \& Padovani 1995), the UV emission of AGN is principally from an accretion disc (Lynden-Bell 1969) surrounding the central SMBH.

We can compare the indices of the variable spectra we measure with those of the average spectra, e.g. those by (Shull et al. 2012) from HST-COS or Vanden Berk et al. (2001), who study quasar spectra over a more similar wavelength range $(1300-5000 \AA)$ to Swift. We find harder indices than either of these measurements. This may be due to our individual dereddening of the sources or there being more fractional variability at shorter wavelengths. An additional constant component which is cooler than the disc would make the total average nuclear spectrum appear redder than the variable part. The consistency of the variable spectra with a flat disc suggests that variable clouds obscuring the disc are unlikely to play a major role at the radii $\left(\sim 50-1000 r_{g}\right)$ probed here.

Contrastingly, we find that the variable X-ray spectra are softer than the average spectra. The X-ray variability is thought to come from fluctuations in the accretion rate in the innermost regions of the disc (e.g. Lyubarskii 1997; King et al. 2004; Zdziarski 2005; Kelly et al. 2011). The softer variable spectra are probably due to the shortest time-scale variations in the harder emission being averaged out across an observation. There may also be a contribution from the continuum varying more than the reflected part, which blurs out variations because it is produced over a larger region. Since the continuum is softer than the reflection it produces, the variable spectrum is softer.

Various groups (e.g. Just et al. 2007; Gibson et al. 2008; Grupe et al. 2010; Vagnetti et al. 2010, 2013) study the relationship between the UV and X-ray average luminosity and find an $\alpha_{\mathrm{OX}}-L_{\mathrm{UV}}$ anticorrelation: brighter UV sources have a lower $L_{\mathrm{X}, \mathrm{Avg}} / L_{\mathrm{UV}, \mathrm{Avg}}$. We find that this is also the case for the variable part of the emission. This means that the fractional variability of UV and X-ray emission behaves similarly between sources, suggesting a link between the two, as expected for an accretion disc and corona system.

\subsection{Sources of variability}

We find that our variable UV spectra are described well by a simple powerlaw. The indices are consistent with those expected for the emission from a disc heated by internal dissipation in the accretion process or by illumination by a central source.

To determine whether the variable UV spectral slope is the result of illumination or a variable accretion disc, we consider the expected time-scales of the two potential variability mechanisms. The shortest time-scales occur towards the centre of the disc, which are principally probed by the higher energy bands. For our fiducial $10^{8} M_{\odot}$ black hole, the shortest wavelength $W^{2}$-band has a half light radius of $\sim 50 r_{g}$. The viscous time-scale at this radius is of the order of 1000 years, so variations in accretion rate due to viscous processes would not occur over the duration of our observations. However, the light-crossing time-scale, which governs variable illumination, is around 1 day and therefore consistent with these observations.

\subsection{X-ray reprocessing}

The variable X-ray emitting corona above the accretion disc directs some of its radiation towards the disc; this is seen both in X-ray reflection spectra (e.g. Tanaka et al. 1995) and X-ray reverberation (e.g. Fabian et al. 2009; Kara et al. 
2016; Alston et al. 2014). If some of this energy is absorbed by the disc, it will be re-emitted thermally in the UV (Lightman \& White 1988). This would suggest a connection between the UV and X-ray emission aside from the feeding of the corona with disc photons.

The correlation between X-ray and UV variability with the UV variations occurring after those in the X-rays in 9 of our sources strongly suggests that X-ray variations drive at least part of the UV variability in those objects. This could be explained by some of the UV variability being due to reprocessing of X-ray radiation.

We find that there is comparable variable power in the $\mathrm{UV}$ and X-ray bands that we measure. While this does not include all of the power in either band, it shows that the Xray variations are sufficiently powerful to drive a significant component of the observed UV variability.

We also note that the X-rays have a level of steady emission: the minimum flux of a given source is typically around one third of the peak flux. If variable illumination is indeed producing notable changes in UV flux, the effect of steady illumination will also be a significant factor in the average flux of the disc.

Further support for the reprocessing scenario comes from the fact that of the 9 sources in which we detect correlated variability, 5 also have iron $\mathrm{K}$ reverberation lags (Kara et al. 2016, 2013b,c). Two of the sources which have measured UV but not iron K lags, Fairall 9 and Ark 120, have relatively high black hole masses $\left(M_{\mathrm{BH}}=8.41\right.$ and 8.18 respectively) so the expected iron $\mathrm{K}$ lags become difficult to detect due to the length of an XMM orbit. Alternatively, the detection of only UV lags may be because the coronal emission is less focussed to the central regions in sources without iron $\mathrm{K}$ lags, so more coronal variable power is delivered to the outer regions of the disc which respond in UV. Equivalently, iron $\mathrm{K}$ reverberation is measured in $1 \mathrm{H} 0707-495$ (Fabian et al. 2009; Zoghbi et al. 2010; Kara et al. 2013a), IC 4329A and PG 1211+143 (both Kara et al. 2016) but we do not find significant correlations, which may reflect a greater proportion of the coronal variability being focussed towards the central regions.

Some previous studies (e.g. Edelson et al. 2015; Troyer et al. 2016; Shappee et al. 2014) have found that the lags in the DCF are longer than the light travel time for a standard thin disc by a factor of a few. Our measurement of the average lag is not sufficiently precise to distinguish between a standard thin disc and these longer measurements. Even where lag times are incompatible with a standard thin disc, there are various explanations for this which still allow for X-ray reprocessing to occur. For example, the perceived disc lags can also be increased by UV emission from emission lines and the Balmer continuum (Korista \& Goad 2001). If these come from the larger BLR, the lags will appear longer. Dexter \& Agol (2011) suggest that local fluctuations in the disc temperature allow hot regions to exist significantly further out than their average radius. These distant hot regions will increase the measured lags while maintaining a thin disc temperature profile on average.

However, the lightcurves are sometimes far from perfectly correlated (for example, the peak correlation coefficient for Fairall 9 is around 0.5), which suggests that at least one of the bands shows variations which do not affect the other. For example, relativistic light bending
(Miniutti \& Fabian 2004) allows the disc to see different variability from a distant observer, particularly if the coronal geometry is changing. It is likely that there are also intrinsic fluctuations in the disc which add to the UV variable power. Variable reddening of the disc emission would also increase the observed UV variable power. The presence of some additional UV variability is supported by Uttley et al. (2003), who find more fractional variability in NGC 5548 in the optical $(5100 \AA$ ) than X-rays in lightcurves binned on a 30 day time-scale.

We therefore conclude that X-ray reprocessing is likely to happen to some extent in all sources and that it is the origin of the observed UV/X-ray lags. However, X-ray reprocessing may not be the only driver of UV variability. Since the viscous time-scale is so much longer than the time-scale of the observed variations, any additional fluctuations must be governed by other processes such as magnetism.

\section{UV/X-RAY CORRELATIONS IN INDIVIDUAL SOURCES - COMPARISON WITH PREVIOUS RESULTS}

\section{$5.1 \quad 1 \mathrm{H} 0707-495$}

In agreement with our results, Robertson et al. (2015) did not find strong correlations between X-ray and W1 band variability in a 7 day long $X M M$ observation, which they ascribe to a particularly compact corona. They found low significance $(\sim 95 \%)$ UV leads, which we also find (at similarly low significance) in the longer lightcurves presented here. These could be due to upscattering of UV emission in the corona. Seemingly at odds with these findings, Xray studies have found strong evidence of iron $\mathrm{K}$ and $\mathrm{L}$ reverberation lags (Fabian et al. 2009; Zoghbi et al. 2010; Kara et al. 2013a) and relativistic reflection (Kara et al. 2015; Dauser et al. 2012) in this source. However, the Swift monitoring includes a period where $1 \mathrm{H} 0707-495$ is in a very low state, and the X-ray reflection is concentrated towards the centre-most region of the disc due to illumination from a low corona $\left(\sim 2 R_{\mathrm{g}}\right)$ (Fabian et al. 2012). The most illuminated region $\left(\lesssim 10 R_{\mathrm{g}}\right)$ is smaller than the region $\left(\sim 500 R_{\mathrm{g}}\right)$ responsible for the bulk of the W2-band emission. This would make UV-X-ray lags hard to detect.

\section{$5.2 \quad$ Fairall 9}

We detect UV lags in all 6 bands, which are largely consistent with the expectations from a thin disc. Recondo-Gonzalez et al. (1997) find variability of up to a factor of $33 \pm 4$ in $I U E$ data of the source, but the mean sampling interval of 96 days is too long to detect lags, giving an upper limit on the lag of $4298 \AA$ behind $1400 \AA$ of 80 days, consistent with our results of lag lengths of a few days. Lohfink et al. (2014) study 2.5 months of Swift monitoring and an $X M M$ observation. They find correlated variability between UV-optical bands on all time-scales measured, down to the Swift sampling time. The additional data now available allow us to show that the longer wavelengths lag the shorter ones. They also find rapid UV flares in the XMM observation, with a lag behind the X-rays of $1-2$ hours at 
$2 \sigma$ significance. We do not have sufficient sampling cadence to verify this result.

\subsection{MCG-6-30-15}

Despite the well-sampled M2-band lightcurve, we are unable to detect lags in MCG-6-30-15. Lira et al. (2015) studied long-term lightcurves in X-rays and optical/near-IR; they found only a weak correlation between X-rays and the $B$ band which does not put useful constraints on the lag. They do find correlations between different optical bands with longer wavelengths lagging shorter ones; these lags are consistent with a $\tau \propto \lambda^{4 / 3}$ relation but for a disc larger than expected by up to a factor of 4 , indicating that weak reprocessing might be taking place. Arévalo et al. (2005) studied $\mathrm{a} \sim 5$ day long $X M M$ observation and found that the $U$ band emission leads the X-rays by $1.9_{-0.8}^{+0.5}$ days, as would be expected for X-rays which are produced by Compton upscattering of UV photons. We would expect to detect such a lag if it were present in the observations analysed here.

While a broad iron line attributed to disc reflection has been detected in the mean X-ray spectrum (Tanaka et al. 1995; Fabian \& Vaughan 2003), Kara et al. (2014) were unable to find an iron $\mathrm{K}$ lag despite having enough counts and variability. They suggest that some variability may be due to geometrical changes in the corona, which would not cause correlated changes in the iron $\mathrm{K}$ emission. Miller et al. (2008) suggest that the red wing of the iron line may be caused by complex absorption, which would not show an iron $\mathrm{K}$ lag. However, using high energy NuSTAR data, Marinucci et al. (2014) favour the relativistic reflection interpretation.

Rapid changes of the geometry of the source could explain the difficulty of detecting X-ray reverberation and reprocessing in the source despite the existence of reflection features in the spectrum.

\subsection{Mrk 335}

We detect a correlation between X-rays and the W2-band but only constrain the lag to $0 \pm 2.9$ days. Grupe et al. (2012) studied the first half of the Swift monitoring used here, finding strong variability but no significant correlation between the X-ray and UV flux. The additional W2 measurements collected after 2012 allow for the detection of a significant correlation now. While we do not determine the direction of the lag, strong reflection (e.g. Parker et al. 2014) indicates that the X-rays illuminate the disc, so some reprocessing is likely to occur and may well be the cause of the correlated variability.

\subsection{Mrk 509}

We do not detect a significant lag in Mrk 509. Since we have only 27 data points for Mrk 509, we would not expect to do so. Marshall et al. (2008) find the optical ( $R$-band) flux leads the X-rays by 15 days in observations from $R X T E$ and ground-based measurements by the SMARTS consortium. Mehdipour et al. (2011) find a correlation between disc and soft X-ray flux in XMM and Swift measurements, which they attribute to warm Comptonisation producing the soft excess.
This interpretation agrees with Boissay et al. (2014), who study spectra from a large XMM / INTEGRAL campaign.

\section{$5.6 \quad$ NGC 3516}

We find a $1.6 \pm 1.5$ day lag of the W2-band behind the X-rays. Edelson et al. (2000) find no significant UV/X-ray correlation in a 3 day observation with HST, RXTE and $A S C A$. Such a short observation is unlikely to detect a lag of the length which we find. Maoz et al. (2002) find a possible 100 day lag of the X-ray relative to the $R$-band but the correlation is not detected in a longer observation (Maoz et al. 2002). They suggest that this may be due to the initial section of the observation being at a higher flux level, in which the X-rays are dominated by a component of emission which does correlate with the UV but is less significant at lower fluxes. Noda et al. (2016) studied observations from Suzaku and Japanese ground-based telescopes. They found a correlation between the hard X-rays and the $B$-band with the X-rays lagging by $2.0_{-0.6}^{+0.7}$ days, which like our measurement is larger than expected for a thin disc.

\subsection{NGC 4051}

The Swift data for NGC 4051 are insufficient in number and frequency to measure the expected lags. While we find a formally significant correlation at 15 days lag, this corresponds to superposing the well sampled ends of the 35 day lightcurves with the unsampled middle of the lightcurve. Peterson et al. (2000) analysed 3 years of $R X T E$ and ground-based observations and found that the long time-scale ( $>30$ day) variability is correlated between optical and X-rays with a lag range of -106 to 68 days. Shemmer et al. (2003) found in 60 days of intensive RXTE monitoring that the DCF centroid showed a UV lead but that the peak may be at a lag, suggesting that both inward propagating fluctuations and X-ray reprocessing are responsible for some of the correlation. Alston et al. (2013) studied the UV/X-ray variability on short time-scales with $X M M$ and found a $3 \mathrm{ks} W 1$-band lag relative to the X-rays. The strength of correlation indicates that $25 \%$ of the UV variance is caused by X-rays. The lag is somewhat shorter than the $\sim 0.2$ day lag detected by Mason et al. (2002) with XMM in the same band at only $85 \%$ confidence. Breedt et al. (2010) correlated 12 years of $R X T E$ observations with $u$ to $I$-band measurements, finding that a $\lambda^{4 / 3}$ relation fits well and that the scaling, subject to significant uncertainties, is consistent with thin disc predictions. As well as lags of a few days, they find lags of $\sim 40$ days which they suggest may be due to the dusty torus which surrounds the inner regions.

\section{$5.8 \quad$ NGC 5548}

We find lags in all bands, increasing with wavelength and always longer than predicted for a thin disc, but consistent within the uncertainties. The STORM campaign has provided an extensive dataset from X-rays to IR: Edelson et al. (2015) measure the UV/X-ray lags against the Hubble $1315 \AA$ band; and (Fausnaugh et al. 2016) extend the wavelength range to the $z$-band $(\sim 9160 \AA)$. This finds that the lags are broadly consistent with $\tau \propto \lambda^{4 / 3}$ but that the disc 
radius is around 3 times the thin-disc prediction. This is in agreement with our findings, which is expected as the majority of Swift data for NGC 5548 is part of the STORM campaign.

\section{$5.9 \quad$ NGC 7469}

We detect significant correlations in 4 UV bands. Our lag measurements are consistent with a thin disc but are poorly constrained. Studying the UV only, Wanders et al. (1997) find delays of UV lines and continuum behind the emission at $1315 \AA$. They find lags increasing with wavelength over 1315-1825 ̊. Collier et al. (1998) find lags between $1315 \AA$ and $4865,6962 \AA$. Kriss et al. (2000) use a HST-FOS spectrum over $1150-3300 \AA$ to better extract spectral bands which are less contaminated by line emission. These measurements of UV continuum lags find that the lags follow a $\lambda^{4 / 3}$ relation. Collier et al. (1999) use these lags to determine $H_{0}$; their value for $H_{0}=42 \pm 9 \mathrm{~km} \mathrm{~s}^{-1} \mathrm{Mpc}^{-1}$ is lower than is now accepted, corresponding to the lags being longer than expected. However, Nandra et al. (1998) find a 4 day UV ( $1315 \AA$ ) lead relative to the X-rays. This could indicate that UV upscattering and X-ray reprocessing are both responsible for some of the correlations.

\subsection{PG $1211+143$}

We find peaks in the DCF at $0 \pm 5$ days, consistent with previous findings, but these are not significant at the $99 \%$ level. Bachev et al. (2009) studied the first section of Swift observations, along with ground-based photometry down to $I$-band. They found lags compatible with a $\lambda^{4 / 3}$ relation at approximately twice the expected lag. Papadakis et al. (2016) also analyse the first Swift section of this dataset but find that the UVOT measurements are consistent with constant flux. Lobban et al. (2016) studied the second section of Swift observations along with XMM-PN/OM data, finding marginally significant X-ray/UV correlations with lags $\lesssim 1$ day.

\section{CONCLUSIONS}

We have presented a variability analysis of archival Swift data from AGN monitoring.

We find that essentially all bands vary and that the variable part of the UV emission has a spectrum consistent with that of the thermal emission from dissipation in an accretion disc or central illumination of a flat disc. The time-scales of variability and lags of UV relative to X-ray variability show that the latter is principally responsible.

The variable power in sources with heavier black holes is higher. The variable UV power increases faster than the variable $\mathrm{X}$-ray power, as is the case for the average emission.

The X-ray and UV variations are significantly correlated in 9 sources; the data for the remaining sources are not sufficient to detect a correlation. All measurements of correlated X-ray/UV variability are consistent with the UV lagging the X-rays. We associate this with the reprocessing of $\mathrm{X}$-rays on the accretion disc.

\section{ACKNOWLEDGEMENTS}

We thank the referee for helpful comments. AL acknowledges useful discussions with Richard Mushotzky. ACF, AML and DJKB acknowledge support from the ERC Advanced Grant FEEDBACK 340442. WNA acknowledges support from the European Union Seventh Framework Programme (FP7/2013-2017) under grant agreement n.312789, StrongGravity. DB acknowledges an STFC studentship. This work made use of data supplied by the UK Swift Science Data Centre at the University of Leicester.

\section{REFERENCES}

Alston W. N., Vaughan S., Uttley P., 2013, MNRAS, 429, 75 Alston W. N., Done C., Vaughan S., 2014, MNRAS, 439, 1548 Antonucci R., 1993, ARA\&A, 31, 473

Arévalo P., Papadakis I., Kuhlbrodt B., Brinkmann W., 2005, A\&A, 430, 435

Ashton C. E., Page M. J., Branduardi-Raymont G., Blustin A. J., 2006, MNRAS, 366, 521

Bachev R., Grupe D., Boeva S., Ovcharov E., Valcheva A., Semkov E., Georgiev T., Gallo L. C., 2009, MNRAS, 399, 750

Bentz M. C., Katz S., 2015, PASP, 127, 67

Bentz M. C., Peterson B. M., Pogge R. W., Vestergaard M., Onken C. A., 2006, ApJ, 644, 133

Bentz M. C., Peterson B. M., Netzer H., Pogge R. W., Vestergaard M., 2009, ApJ, 697, 160

Bianchi S., Guainazzi M., Matt G., Fonseca Bonilla N., Ponti G., 2009, A\&A, 495, 421

Boissay R., et al., 2014, A\&A, 567, A44

Breedt E., et al., 2010, MNRAS, 403, 605

Burrows D. N., et al., 2005, Space Sci. Rev., 120, 165

Cackett E. M., Horne K., Winkler H., 2007, MNRAS, 380, 669

Cameron D. T., McHardy I., Dwelly T., Breedt E., Uttley P., Lira P., Arevalo P., 2012, MNRAS, 422, 902

Cardelli J. A., Clayton G. C., Mathis J. S., 1989, ApJ, 345, 245

Chartas G., et al., 2016, Astronomische Nachrichten, 337, 356

Collier S. J., et al., 1998, ApJ, 500, 162

Collier S., Horne K., Wanders I., Peterson B. M., 1999, MNRAS, 302, L24

Dauser T., et al., 2012, MNRAS, 422, 1914

Davis S. W., Woo J.-H., Blaes O. M., 2007, ApJ, 668, 682

Dexter J., Agol E., 2011, ApJ, 727, L24

Edelson R. A., Krolik J. H., 1988, ApJ, 333, 646

Edelson R., et al., 2000, ApJ, 534, 180

Edelson R., Turner T. J., Pounds K., Vaughan S., Markowitz A., Marshall H., Dobbie P., Warwick R., 2002, ApJ, 568, 610

Edelson R., et al., 2015, ApJ, 806, 129

Evans P. A., et al., 2007, A\&A, 469, 379

Evans P. A., et al., 2009, MNRAS, 397, 1177

Fabian A. C., Vaughan S., 2003, MNRAS, 340, L28

Fabian A. C., et al., 2009, Nature, 459, 540

Fabian A. C., et al., 2012, MNRAS, 419, 116

Fausnaugh M. M., et al., 2016, ApJ, 821, 56

Francis P. J., Hewett P. C., Foltz C. B., Chaffee F. H., Weymann R. J., Morris S. L., 1991, ApJ, 373, 465

Gaskell C. M., Klimek E. S., 2003, Astronomical and Astrophysical Transactions, 22, 661

Gaskell C. M., Peterson B. M., 1987, ApJS, 65, 1

Gaskell C. M., Sparke L. S., 1986, ApJ, 305, 175

Gehrels N., et al., 2004, ApJ, 611, 1005

Gibson R. R., Brandt W. N., Schneider D. P., 2008, ApJ, 685, 773

González-Martín O., Vaughan S., 2012, A\&A, 544, A80

Grupe D., Komossa S., Leighly K. M., Page K. L., 2010, ApJS, 187,64 
Grupe D., Komossa S., Gallo L. C., Lia Longinotti A., Fabian A. C., Pradhan A. K., Gruberbauer M., Xu D., 2012, ApJS, 199,28

Haardt F., Maraschi L., 1991, ApJ, 380, L51

Hjorth J., Vestergaard M., Sorensen A. N., Grundahl F., 1995, ApJ, 452, L17

Just D. W., Brandt W. N., Shemmer O., Steffen A. T., Schneider D. P., Chartas G., Garmire G. P., 2007, ApJ, 665, 1004

Kalberla P. M. W., Burton W. B., Hartmann D., Arnal E. M., Bajaja E., Morras R., Pöppel W. G. L., 2005, A\&A, 440, 775

Kara E., Fabian A. C., Cackett E. M., Steiner J. F., Uttley P., Wilkins D. R., Zoghbi A., 2013a, MNRAS, 428, 2795

Kara E., Fabian A. C., Cackett E. M., Miniutti G., Uttley P., 2013b, MNRAS, 430, 1408

Kara E., Fabian A. C., Cackett E. M., Uttley P., Wilkins D. R., Zoghbi A., 2013c, MNRAS, 434, 1129

Kara E., et al., 2014, MNRAS, 445, 56

Kara E., et al., 2015, MNRAS, 449, 234

Kara E., Alston W., Fabian A., 2016, Astronomische Nachrichten, 337,473

Kelly B. C., Sobolewska M., Siemiginowska A., 2011, ApJ, 730, 52

King A. R., Pringle J. E., West R. G., Livio M., 2004, MNRAS, 348,111

Kishimoto M., Hönig S. F., Antonucci R., Millour F., Tristram K. R. W., Weigelt G., 2011, A\&A, 536, A78

Korista K. T., Goad M. R., 2001, ApJ, 553, 695

Kriss G. A., Peterson B. M., Crenshaw D. M., Zheng W., 2000, ApJ, 535, 58

Krolik J. H., Kallman T. R., 1988, ApJ, 324, 714

Lightman A. P., White T. R., 1988, ApJ, 335, 57

Lira P., Arévalo P., Uttley P., McHardy I. M. M., Videla L., 2015, MNRAS, 454, 368

Lobban A. P., Vaughan S., Pounds K., Reeves J. N., 2016 , MNRAS, 457, 38

Lohfink A. M., Reynolds C. S., Vasudevan R., Mushotzky R. F., Miller N. A., 2014, ApJ, 788, 10

Lusso E., Risaliti G., 2016, ApJ, 819, 154

Lynden-Bell D., 1969, Nature, 223, 690

Lyubarskii Y. E., 1997, MNRAS, 292, 679

Maoz D., Edelson R., Nandra K., 2000, AJ, 119, 119

Maoz D., Markowitz A., Edelson R., Nandra K., 2002, AJ, 124,1988

Marinucci A., et al., 2014, ApJ, 787, 83

Markowitz A., 2009, ApJ, 698, 1740

Marshall K., Ryle W. T., Miller H. R., 2008, ApJ, 677, 880

Marziani P., Calvani M., Sulentic J. W., 1992, ApJ, 393, 658

Mason K. O., et al., 2002, ApJ, 580, L117

McHardy I. M., 2013, MNRAS, 430, L49

McHardy I. M., et al., 2016, Astronomische Nachrichten, 337, 500

Mehdipour M., et al., 2011, A\&A, 534, A39

Meléndez M., Kraemer S. B., Weaver K. A., Mushotzky R. F., 2011, ApJ, 738, 6

Miller L., Turner T. J., Reeves J. N., 2008, A\&A, 483, 437

Miniutti G., Fabian A. C., 2004, MNRAS, 349, 1435

Morgan C. W., Kochanek C. S., Morgan N. D., Falco E. E., 2010, ApJ, 712, 1129

Mushotzky R. F., Done C., Pounds K. A., 1993, ARA\&A, 31, 717

Nandra K., George I. M., Mushotzky R. F., Turner T. J., Yaqoob T., 1997, ApJ, 476, 70

Nandra K., Clavel J., Edelson R. A., George I. M., Malkan M. A., Mushotzky R. F., Peterson B. M., Turner T. J., 1998, ApJ, 505,594

Noda H., et al., 2016, preprint, (arXiv:1605.08050)

O'Donnell J. E., 1994, ApJ, 422, 158

Pan H.-W., Yuan W., Yao S., Zhou X.-L., Liu B., Zhou H., Zhang S.-N., 2016, ApJ, 819, L19

Papadakis I. E., Nicastro F., Panagiotou C., 2016, A\&A, 591, A102
Parker M. L., et al., 2014, MNRAS, 443, 1723

Pei Y. C., 1992, ApJ, 395, 130

Peterson B. M., Wanders I., Horne K., Collier S., Alexander T., Kaspi S., Maoz D., 1998, PASP, 110, 660

Peterson B. M., et al., 2000, ApJ, 542, 161

Peterson B. M., et al., 2004, ApJ, 613, 682

Polletta M., Courvoisier T. J.-L., 1999, A\&A, 350, 765

Poole T. S., et al., 2008, MNRAS, 383, 627

Poutanen J., Zdziarski A. A., Ibragimov A., 2008, MNRAS, 389,1427

Pringle J. E., 1981, ARA\&A, 19, 137

Recondo-Gonzalez M. C., Wamsteker W., Clavel J., RodriguezPascual P. M., Vio R., Ting-Gui W., Santos-Lleo M., Makino F., 1997, A\&AS, 121

Robertson D. R. S., Gallo L. C., Zoghbi A., Fabian A. C., 2015 , MNRAS, 453, 3455

Roming P. W. A., et al., 2005, Space Sci. Rev., 120, 95

Schneider D. P., Schmidt M., Gunn J. E., 1991, AJ, 101, 2004

Shakura N. I., Sunyaev R. A., 1973, A\&A, 24, 337

Shappee B. J., et al., 2014, ApJ, 788, 48

Shemmer O., et al., 2001, ApJ, 561, 162

Shemmer O., Uttley P., Netzer H., McHardy I. M., 2003, MNRAS, 343,1341

Shemmer O., Brandt W. N., Netzer H., Maiolino R., Kaspi S., 2008, ApJ, 682, 81

Shull J. M., Stevans M., Danforth C. W., 2012, ApJ, 752, 162

Smith R., Vaughan S., 2007, MNRAS, 375, 1479

Steffen A. T., Strateva I., Brandt W. N., Alexander D. M., Koekemoer A. M., Lehmer B. D., Schneider D. P., Vignali C., 2006, AJ, 131, 2826

Strateva I. V., Brandt W. N., Schneider D. P., Vanden Berk D. G., Vignali C., 2005, AJ, 130, 387

Tanaka Y., et al., 1995, Nature, 375, 659

Telfer R. C., Zheng W., Kriss G. A., Davidsen A. F., 2002, ApJ, 565,773

Timmer J., Koenig M., 1995, A\&A, 300, 707

Trevese D., Perna M., Vagnetti F., Saturni F. G., Dadina M., 2014, ApJ, 795, 164

Troyer J., Starkey D., Cackett E. M., Bentz M. C., Goad M. R., Horne K., Seals J. E., 2016, MNRAS, 456, 4040

Urry C. M., Padovani P., 1995, PASP, 107, 803

Uttley P., Edelson R., McHardy I. M., Peterson B. M., Markowitz A., 2003, ApJ, 584, L53

Uttley P., McHardy I. M., Vaughan S., 2005, MNRAS, 359, 345

Uttley P., Cackett E. M., Fabian A. C., Kara E., Wilkins D. R., 2014, A\&ARv, 22, 72

Vagnetti F., Turriziani S., Trevese D., Antonucci M., 2010, A\&A, 519, A17

Vagnetti F., Antonucci M., Trevese D., 2013, A\&A, 550, A71

Vanden Berk D. E., et al., 2001, AJ, 122, 549

Vasudevan R. V., Fabian A. C., 2007, MNRAS, 381, 1235

Vasudevan R. V., Fabian A. C., 2009, MNRAS, 392, 1124

Vasudevan R. V., Fabian A. C., Gandhi P., Winter L. M., Mushotzky R. F., 2010, MNRAS, 402, 1081

Vaughan S., Edelson R., Warwick R. S., Uttley P., 2003, MNRAS, 345,1271

Vaughan S., Uttley P., Pounds K. A., Nandra K., Strohmayer T. E., 2011, MNRAS, 413, 2489

Vestergaard M., 2002, ApJ, 571, 733

Vignali C., Brandt W. N., Schneider D. P., 2003, AJ, 125, 433

Wanders I., et al., 1997, ApJS, 113, 69

Ward M., Elvis M., Fabbiano G., Carleton N. P., Willner S. P., Lawrence A., 1987, ApJ, 315, 74

Welsh W. F., 1999, PASP, 111, 1347

Winter L. M., Lewis K. T., Koss M., Veilleux S., Keeney B., Mushotzky R. F., 2010, ApJ, 710, 503

Xu Y.-D., 2011, ApJ, 739, 64

Zdziarski A. A., 2005, MNRAS, 360, 816 
14 D. J. K. Buisson et al.

Zhou X.-L., Wang J.-M., 2005, ApJ, 618, L83

Zoghbi A., Fabian A. C., Uttley P., Miniutti G., Gallo L. C.,

Reynolds C. S., Miller J. M., Ponti G., 2010, MNRAS,

401, 2419 


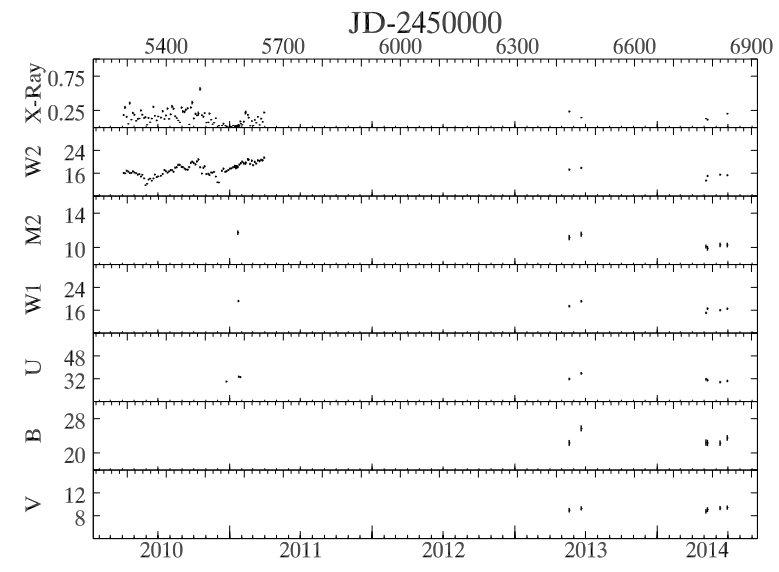

Figure A1. 1H 0707-495

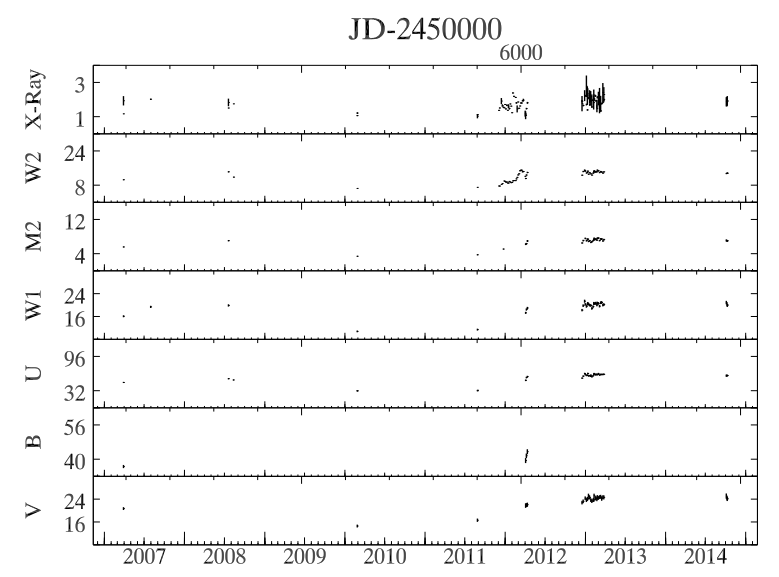

Figure A2. 3C 120

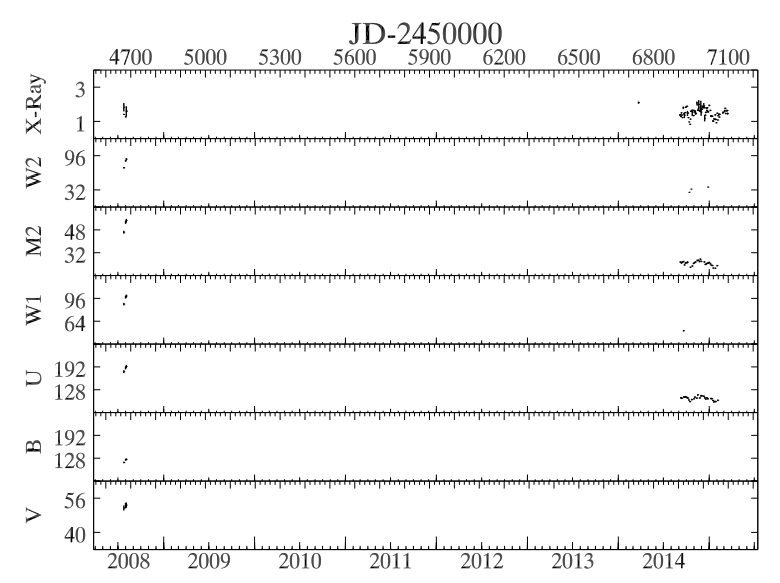

Figure A3. ARK 120

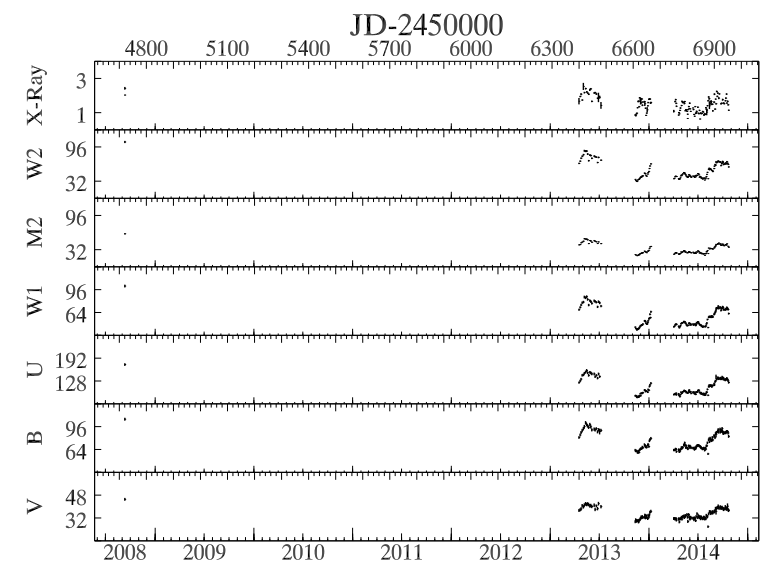

Figure A4. Fairall 9

\section{APPENDIX A: LIGHTCURVES}

Lightcurves for each source in our sample in each band.

Rates are given in $\mathrm{cts} \mathrm{s}^{-1}$; X-rays are measured over 0.3 -

$10 \mathrm{keV}$ 


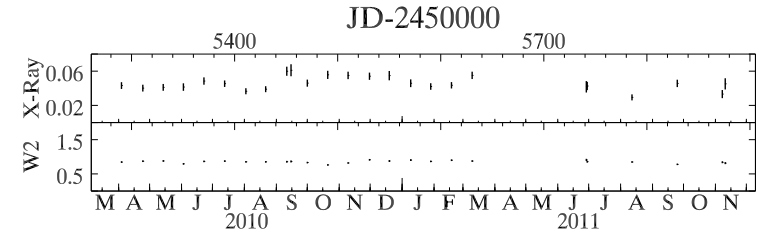

Figure A5. H 0557-385

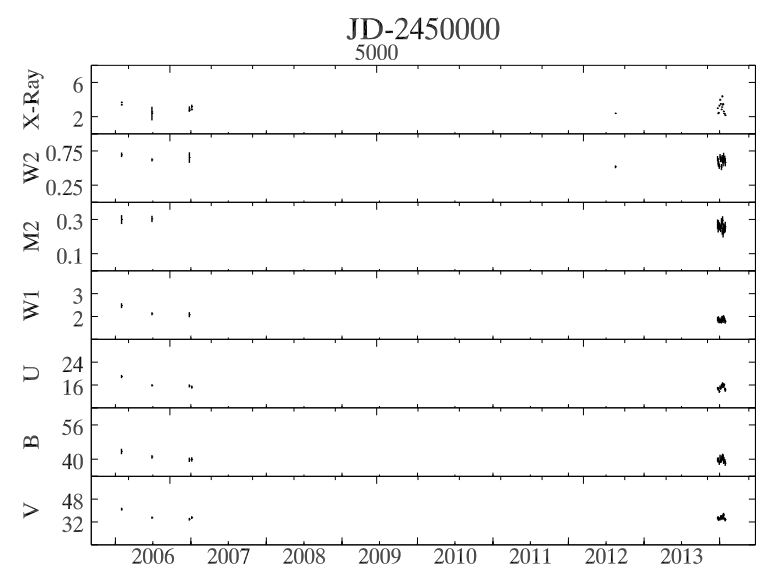

Figure A6. IC 4329A

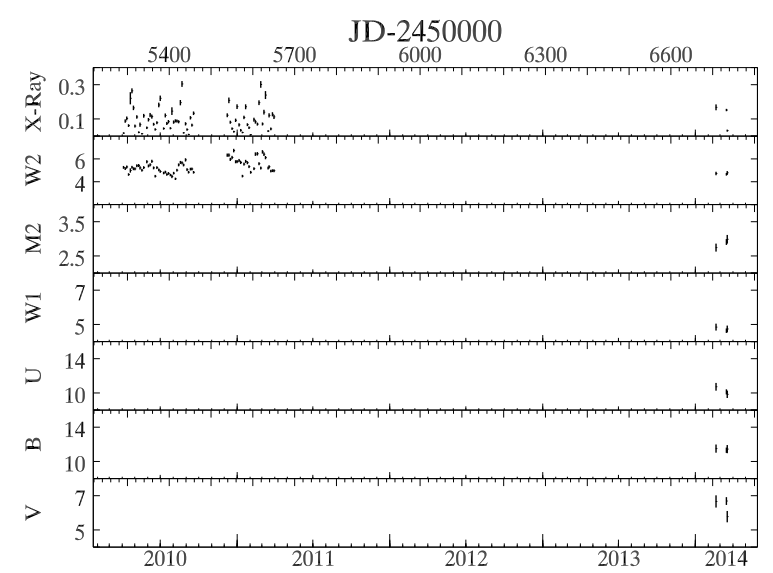

Figure A7. IRAS 13224-3809

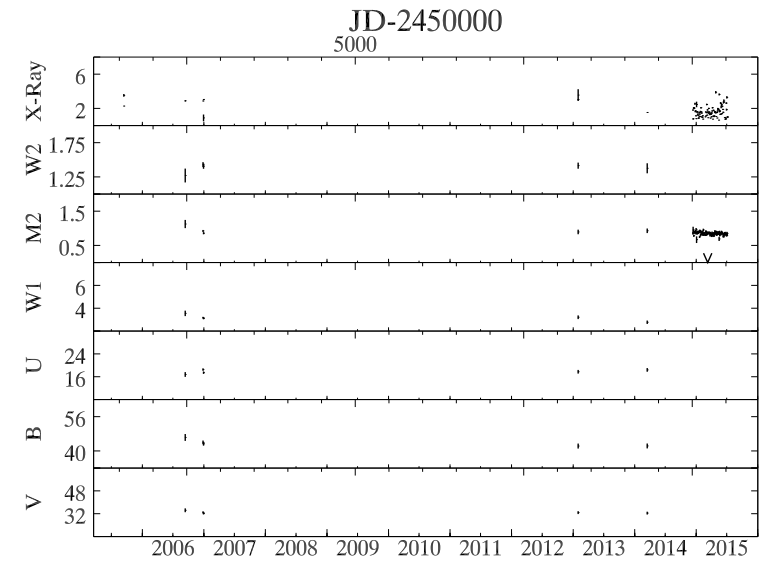

Figure A8. MCG-6-30-15

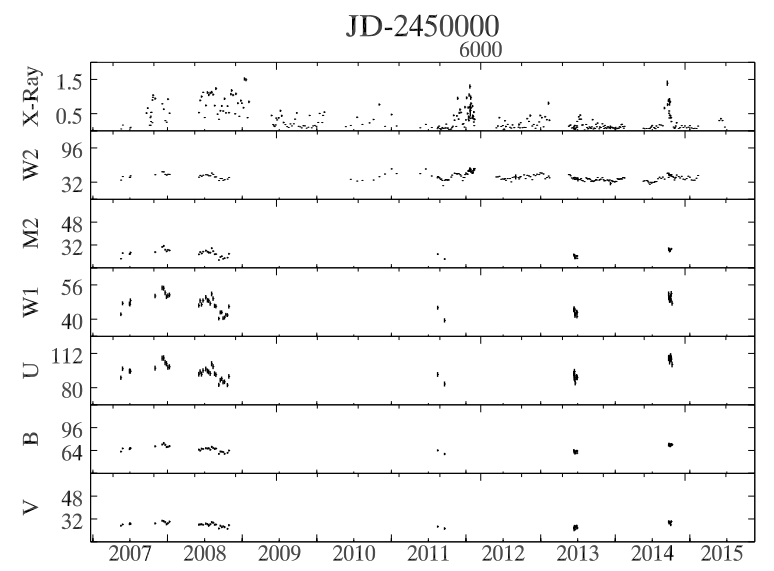

Figure A9. MRK 335

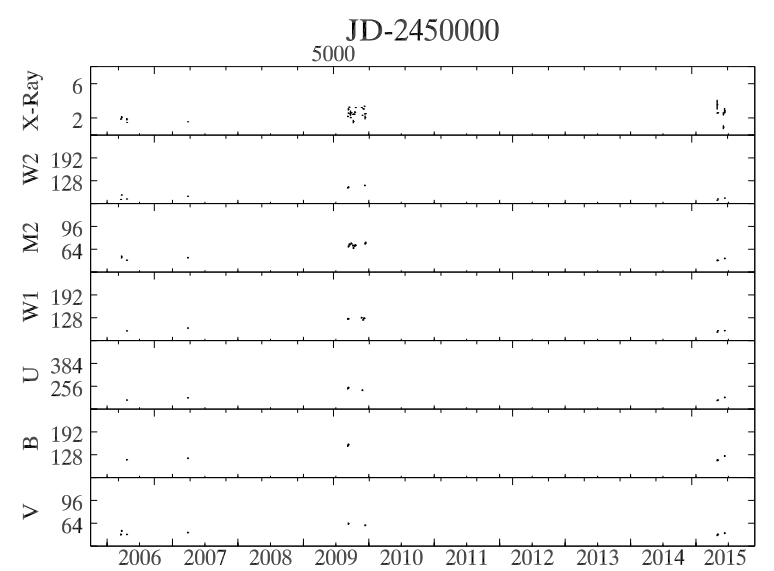

Figure A10. MRK 509 
JD-2450000

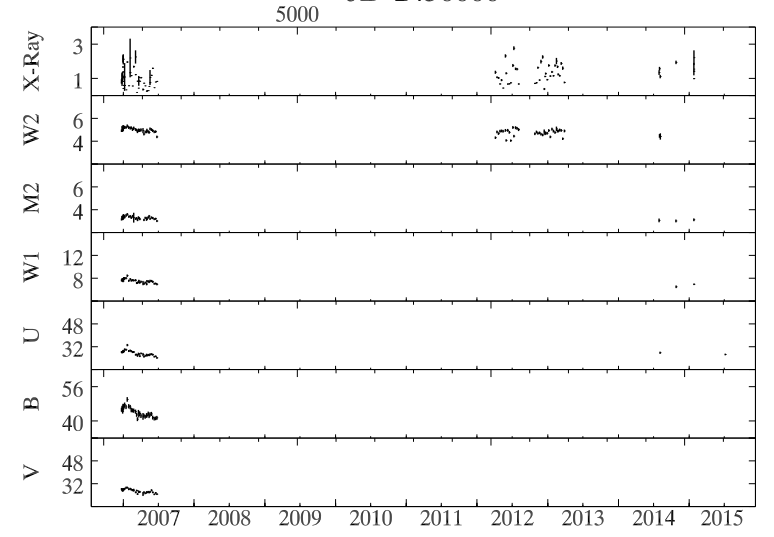

Figure A11. MRK 766

JD-2450000

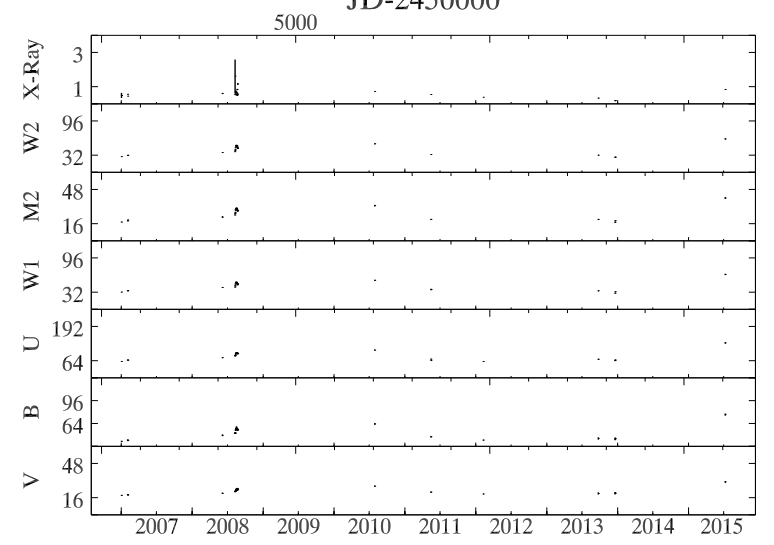

Figure A12. MRK 841

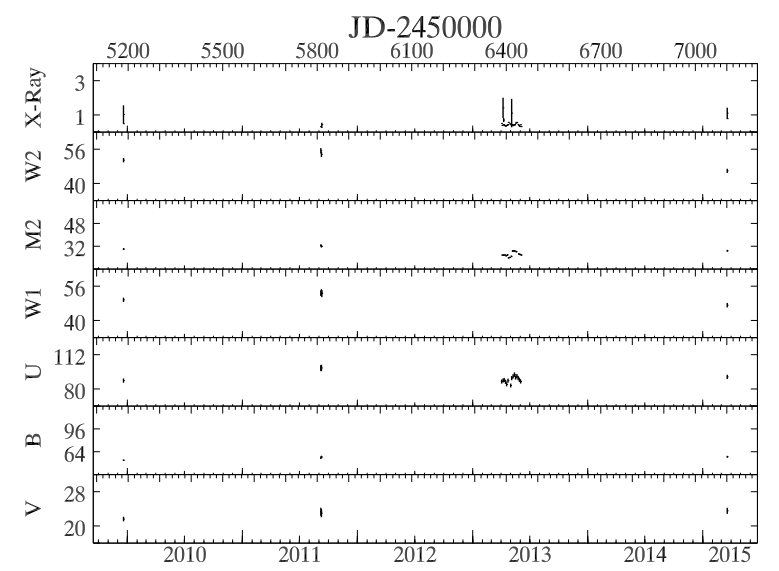

Figure A13. MRK 1383
JD-2450000

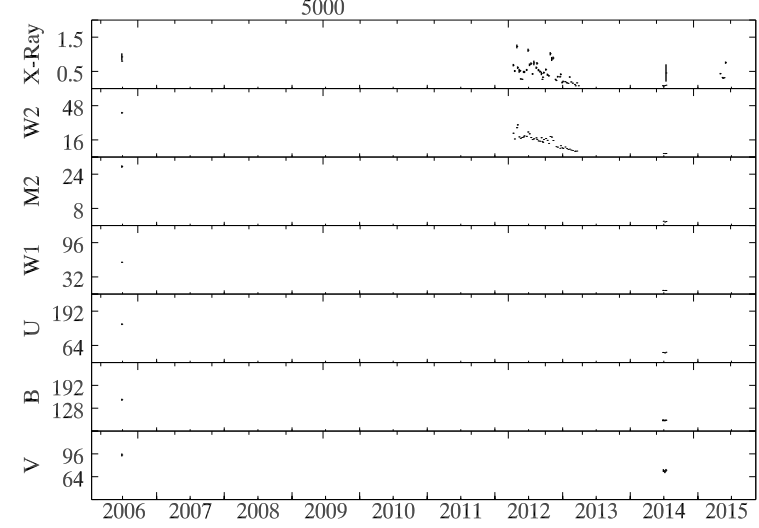

Figure A14. NGC 3516

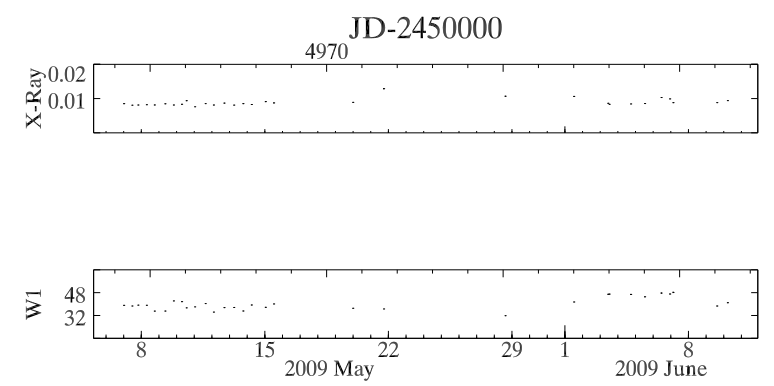

Figure A15. NGC 4051

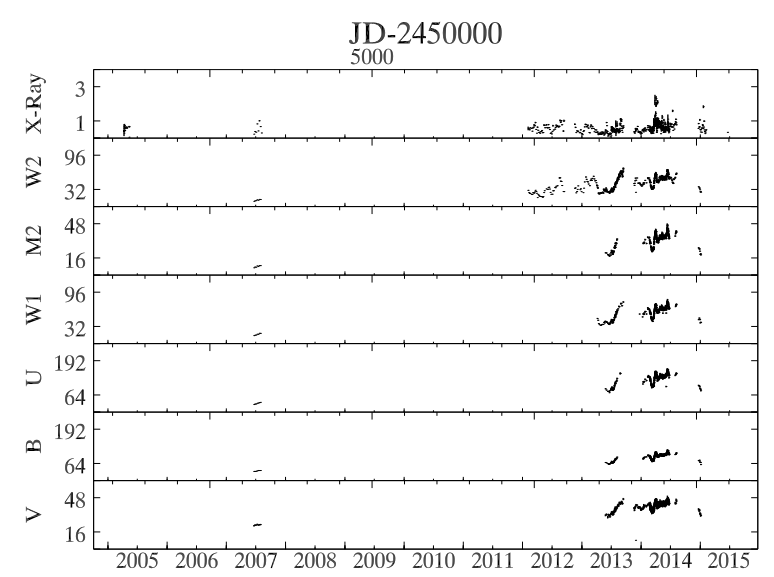

Figure A16. NGC 5548 


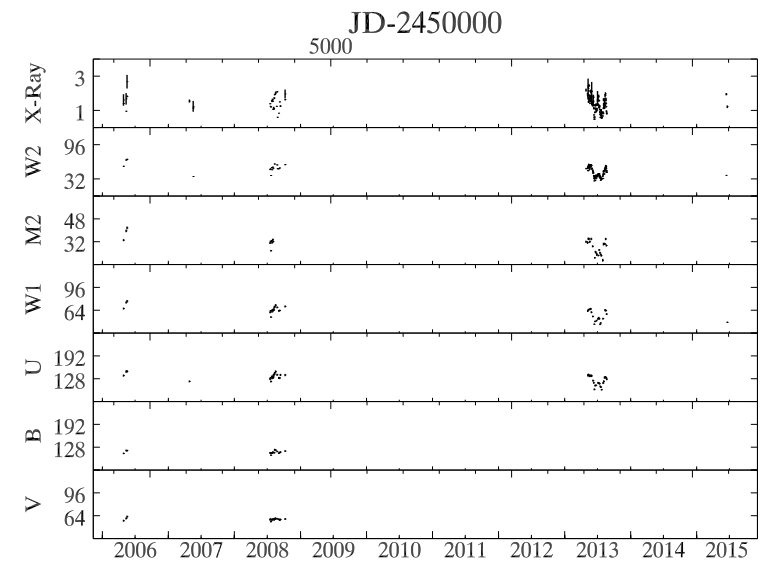

Figure A17. NGC 7469
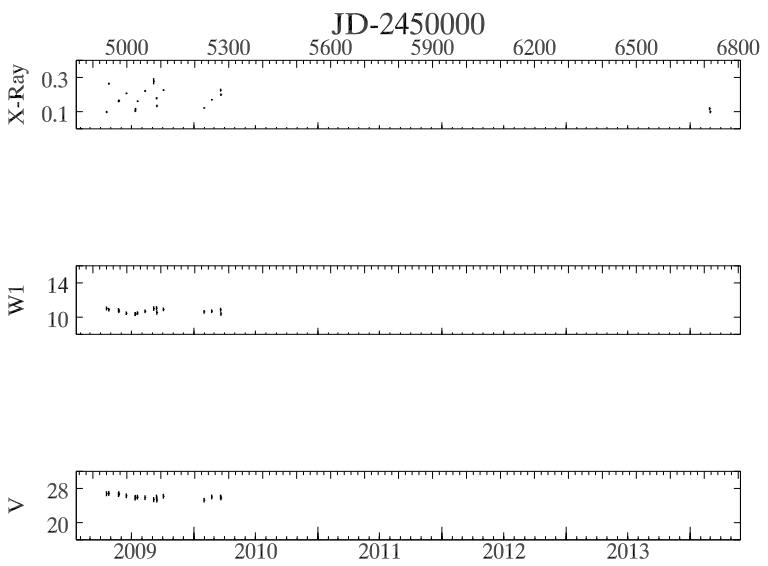

Figure A18. PDS 456

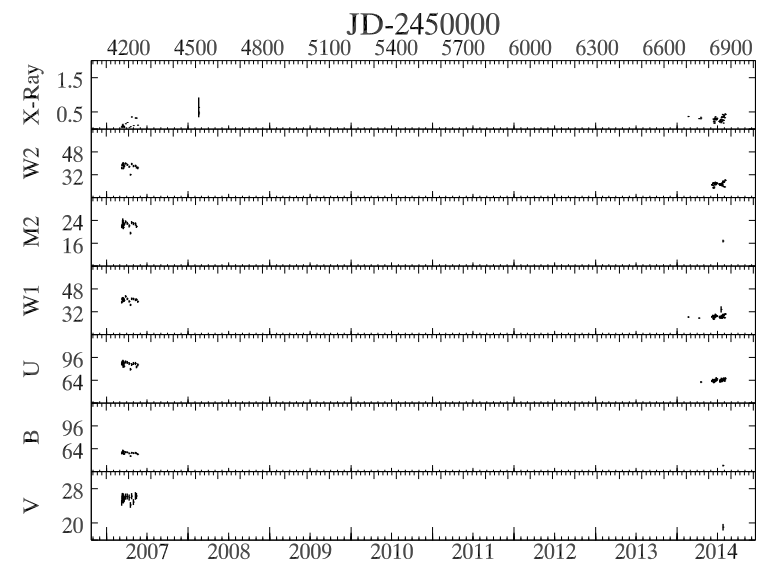

Figure A19. PG $1211+143$

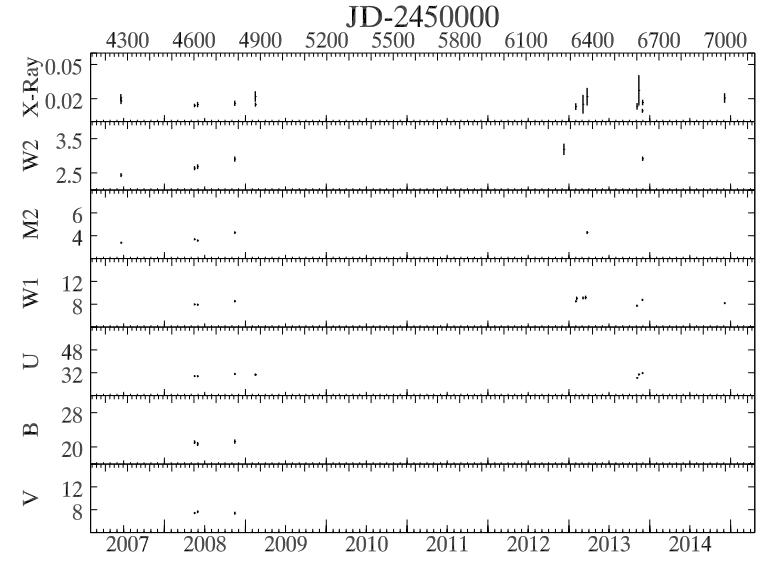

Figure A20. PG $1247+267$

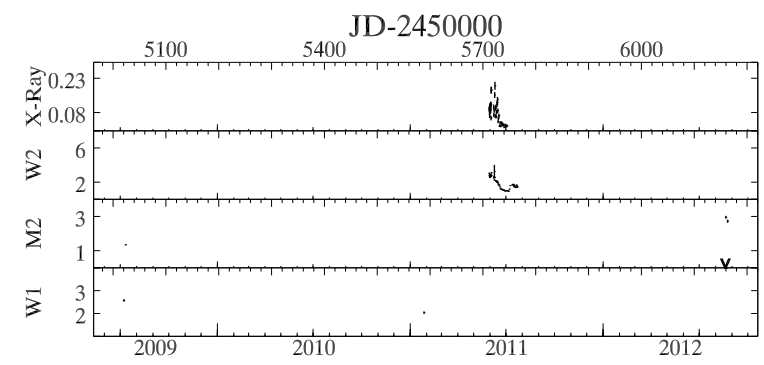

Figure A21. Zw229-15 


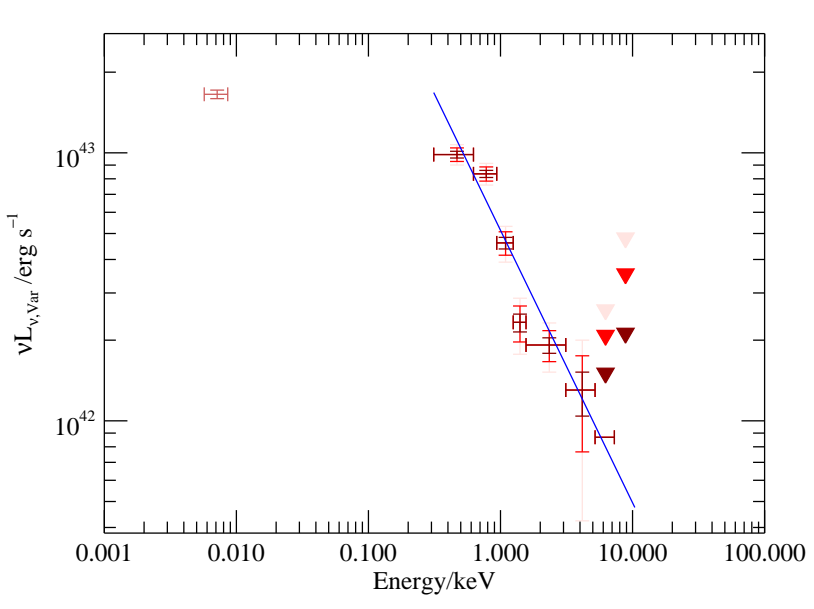

Figure B1. 1H 0707-495

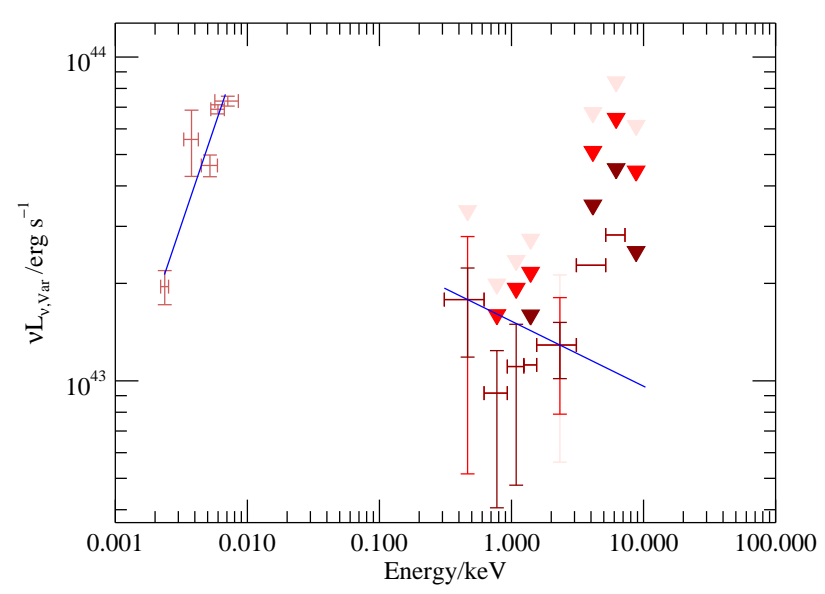

Figure B2. 3C 120

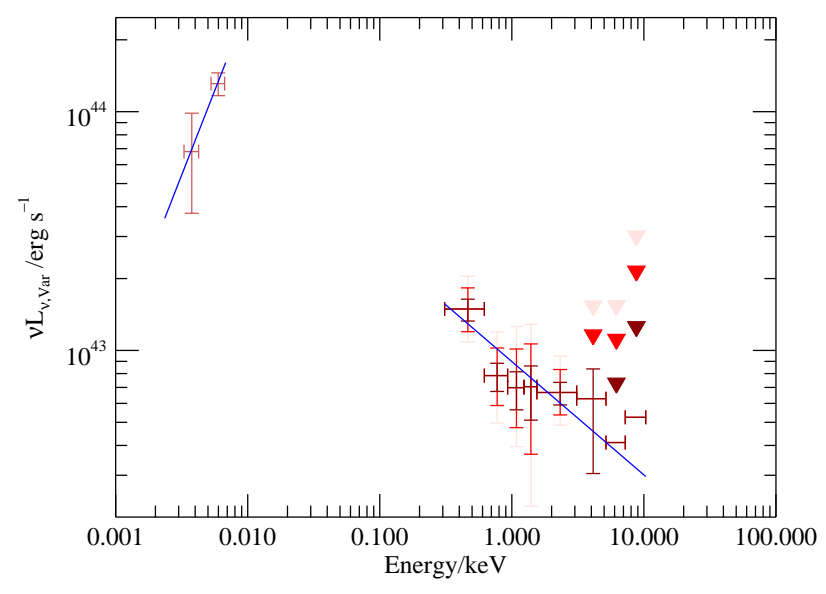

Figure B3. ARK 120

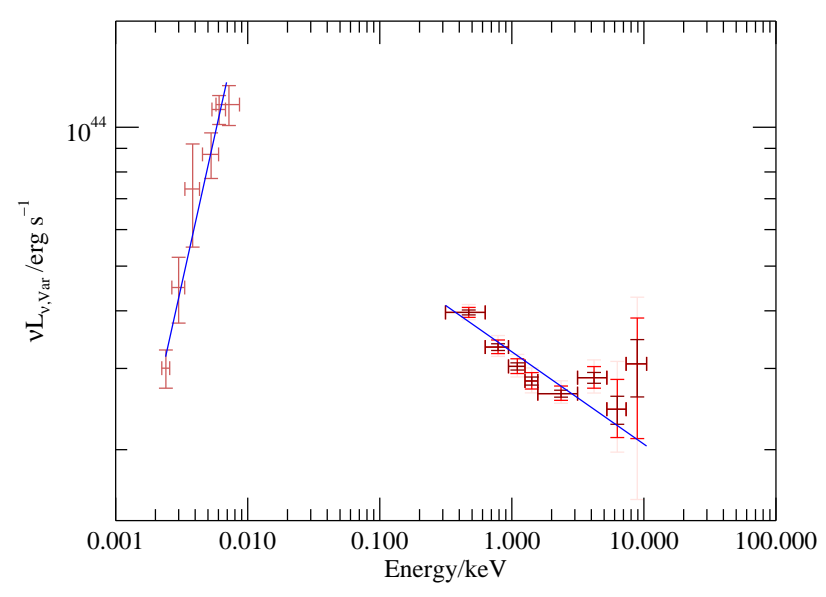

Figure B4. Fairall 9

\section{APPENDIX B: BROADBAND VARIABILITY \\ SPECTRA}

Energies are given in the source frame. UV errors are shown at 1- $\sigma$. 1,2 and 3- $\sigma$ X-ray errors are shown in maroon, red and pink respectively; triangles represent upper limits. Blue lines show powerlaw fits to UV and X-ray bands separately; those for X-rays only include points detected at $2-\sigma$. 
20

D. J. K. Buisson et al.

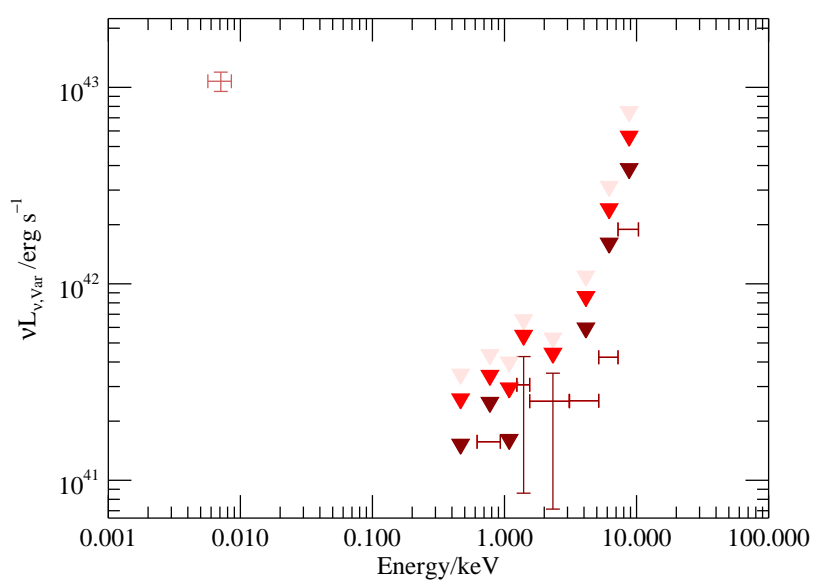

Figure B5. H 0557-385

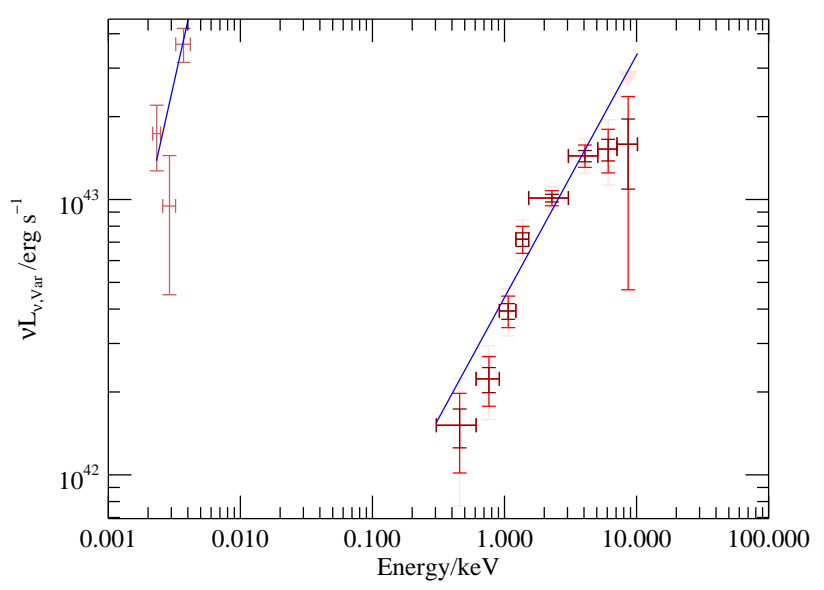

Figure B6. IC 4329A

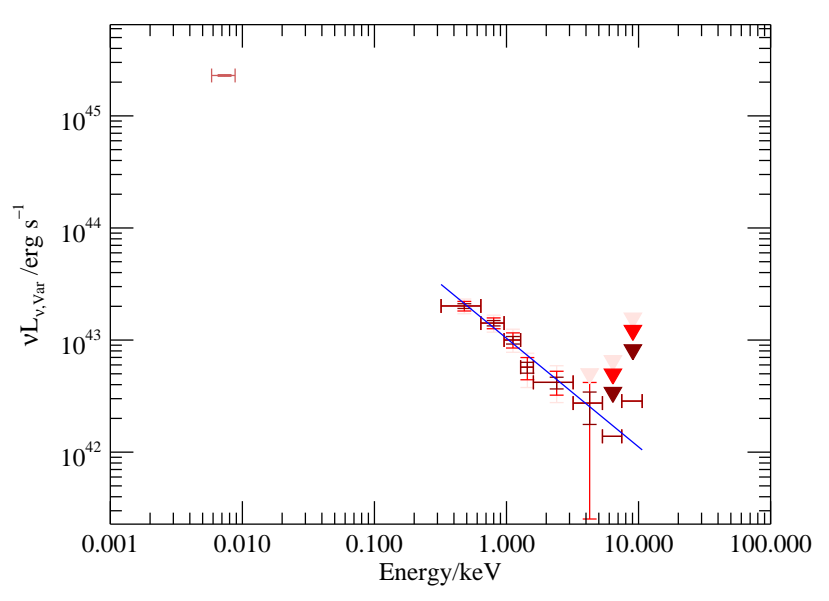

Figure B7. IRAS 13224-3809

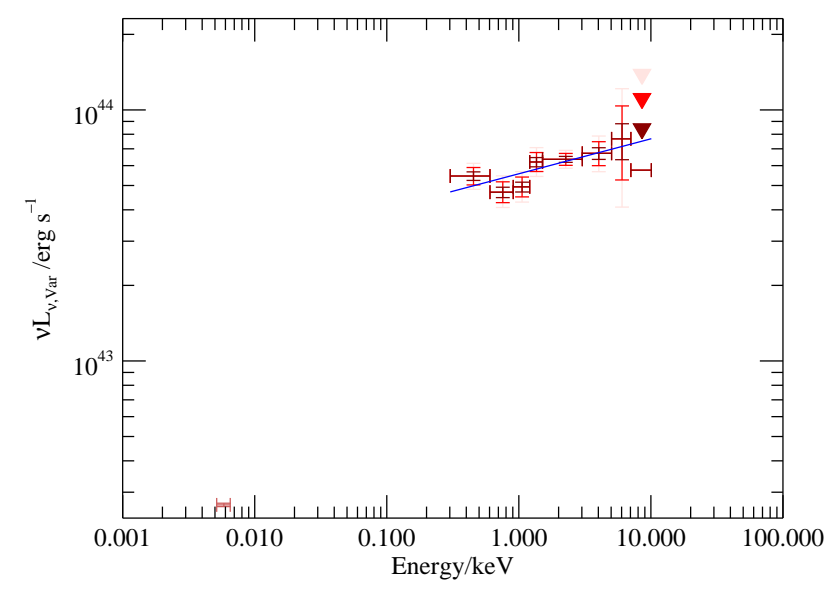

Figure B8. MCG-6-30-15

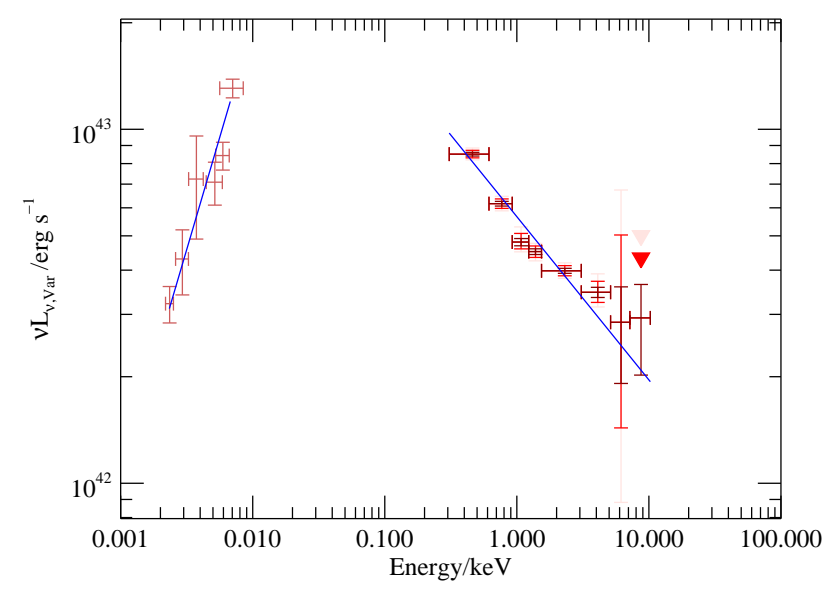

Figure B9. MRK 335

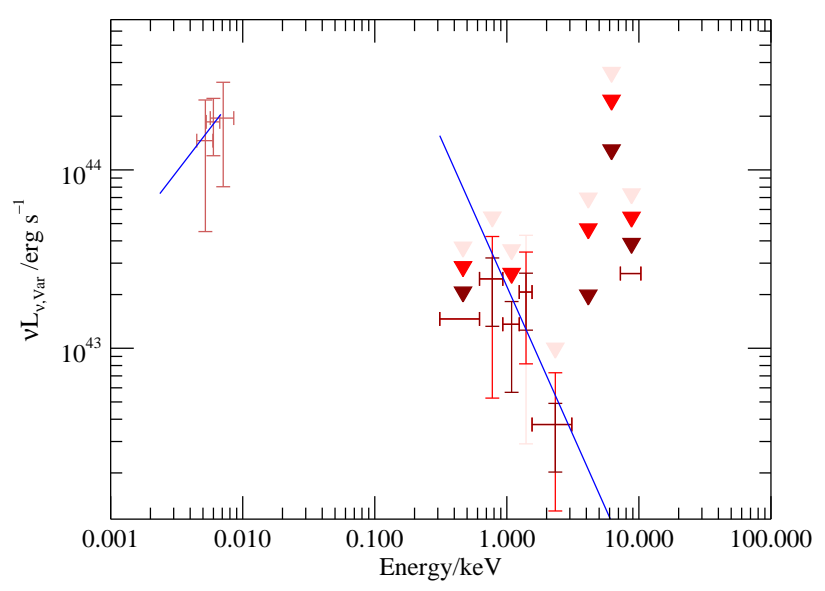

Figure B10. MRK 509

MNRAS 000, 1-28 (2016) 
$U V$ and $X$-ray variability of $A G N$ with Swift

21

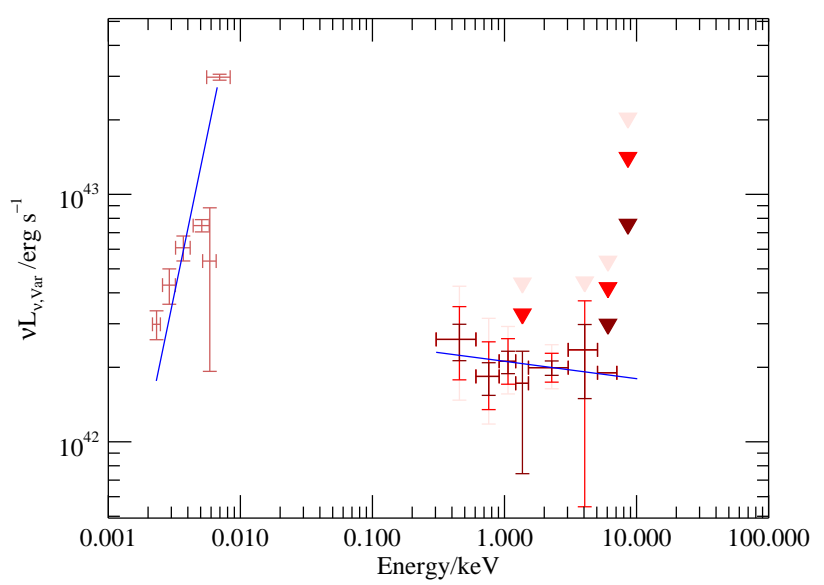

Figure B11. MRK 766

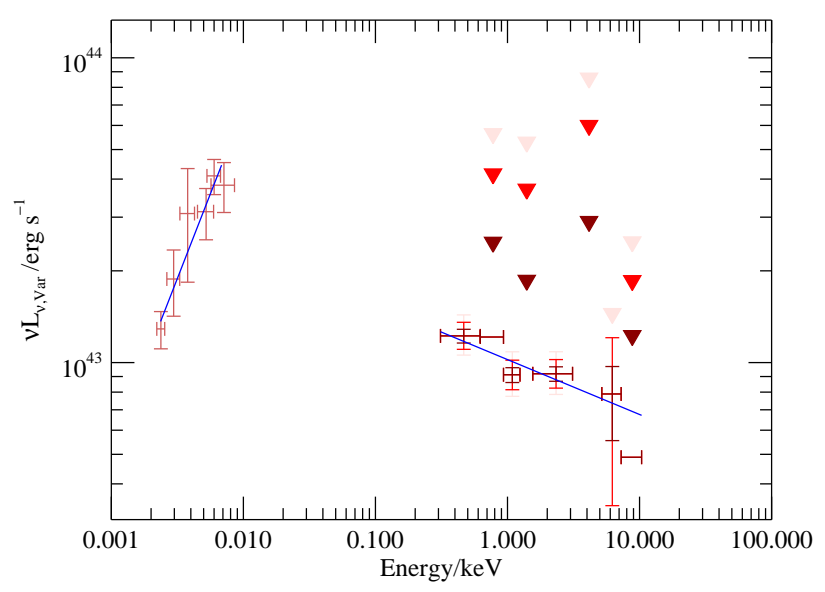

Figure B12. MRK 841

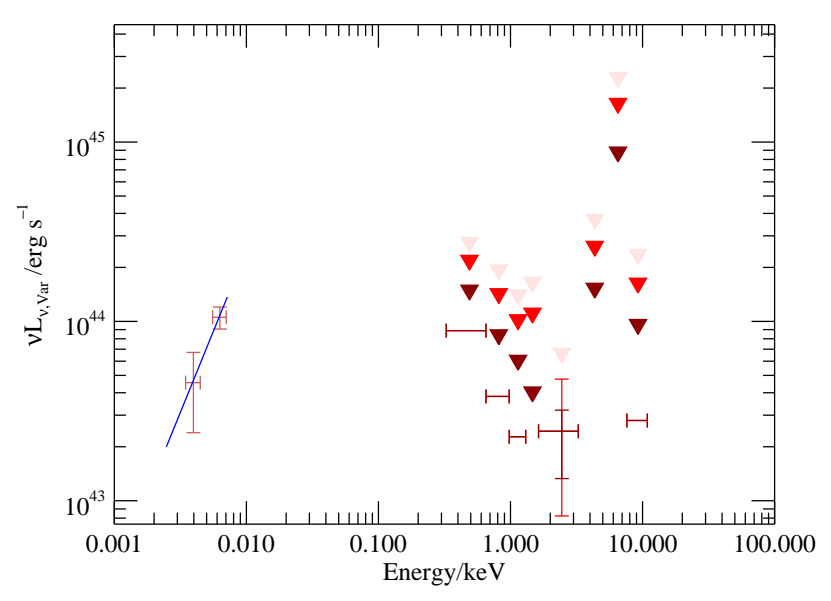

Figure B13. MRK 1383

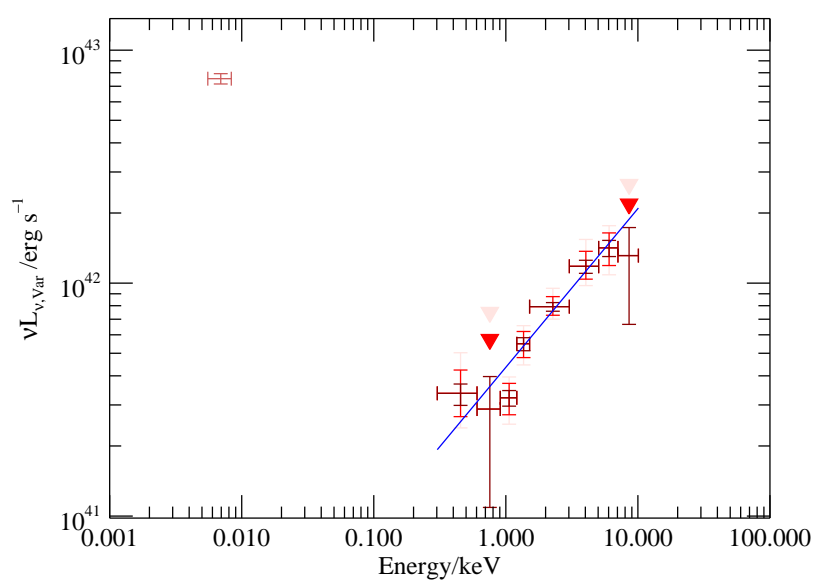

Figure B14. NGC 3516

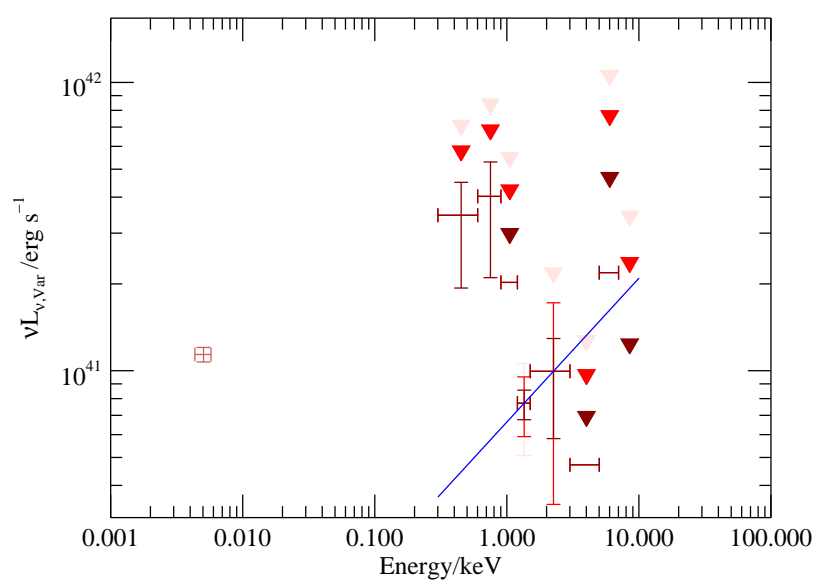

Figure B15. NGC 4051

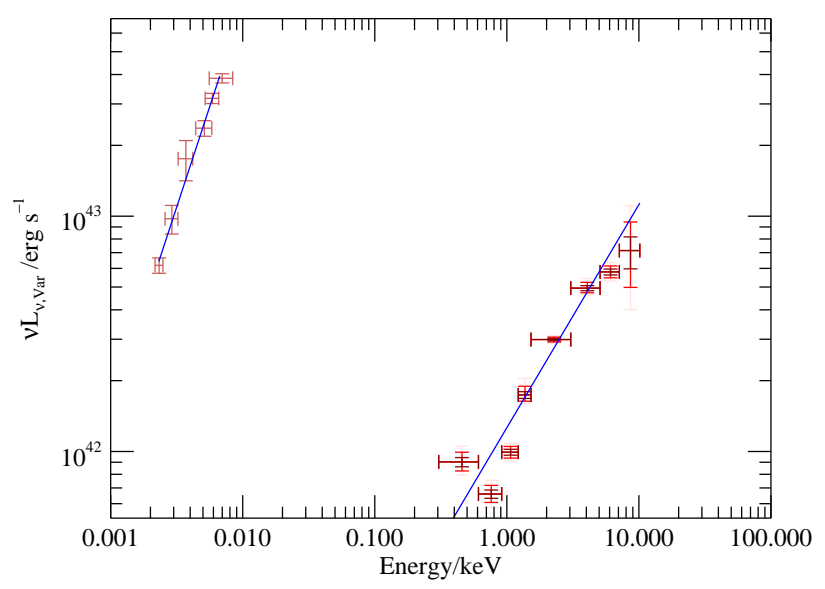

Figure B16. NGC 5548

MNRAS 000, 1-28 (2016) 
22

D. J. K. Buisson et al.

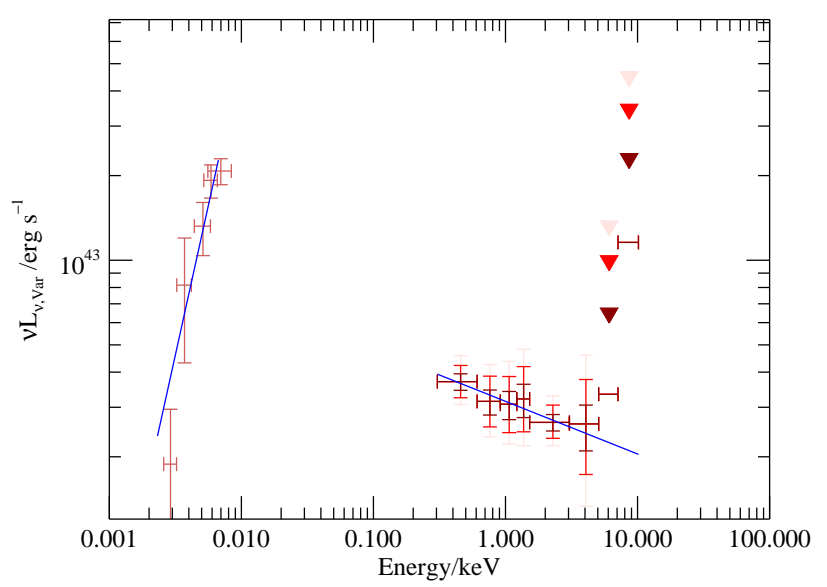

Figure B17. NGC 7469

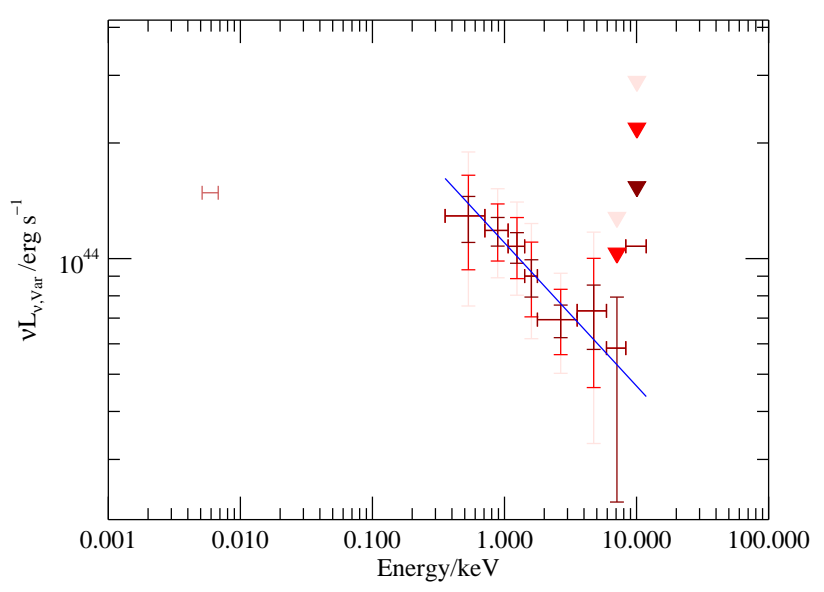

Figure B18. PDS 456

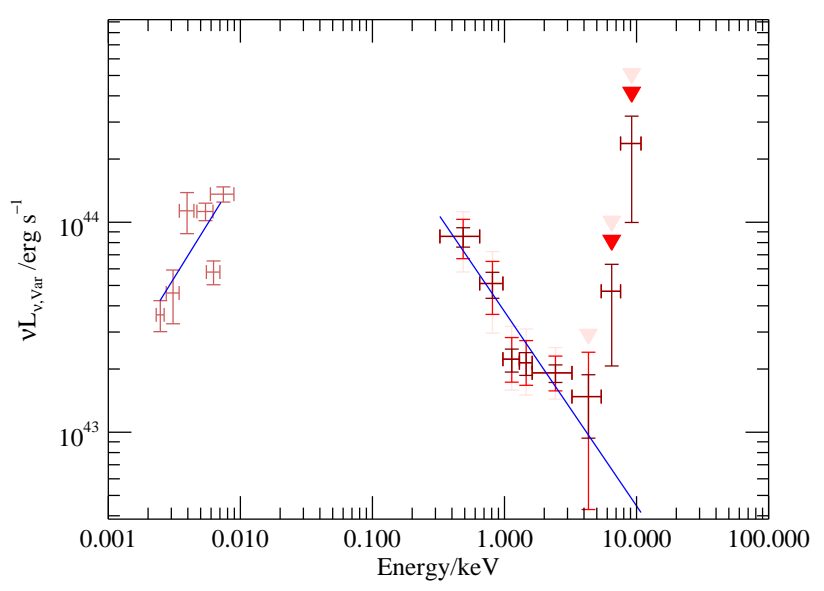

Figure B19. PG 1211+143

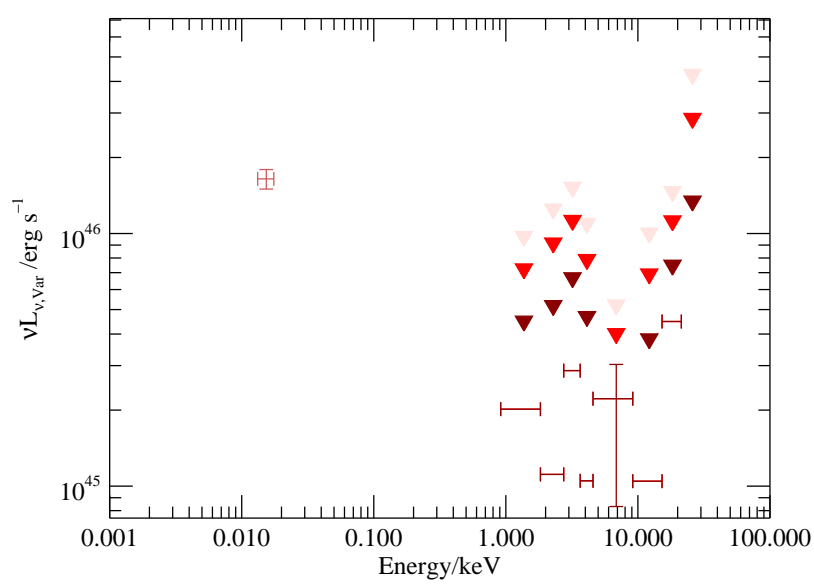

Figure B20. PG $1247+267$

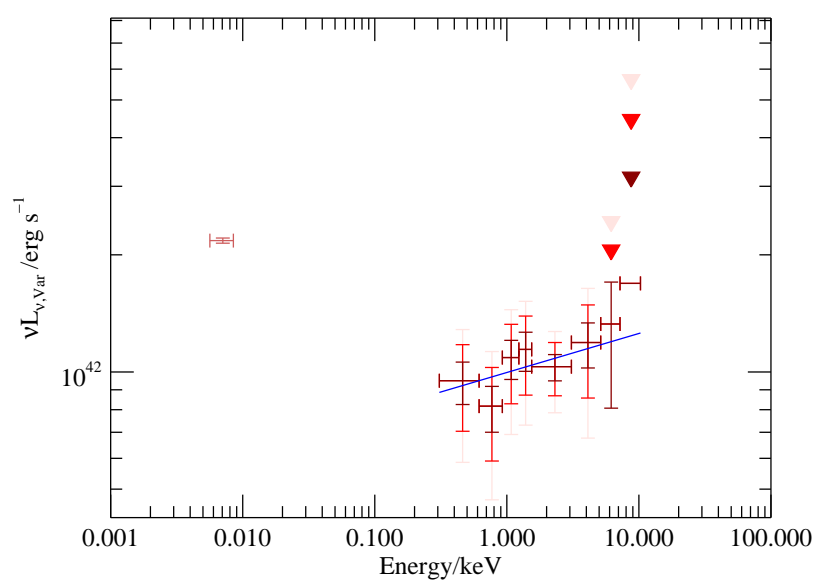

Figure B21. Zw 229-15

MNRAS 000, 1-28 (2016) 


\section{APPENDIX C: DCFS}

Discrete correlation function (black) of given band relative to X-rays. Positive lag indicates UV variations occurring after X-ray variations. 95 and $99 \%$ confidence intervals are shown in blue and red respectively. The ACF of the Xrays and UV are shown in green dashed and purple dotted lines respectively. Plots with fewer than 3 DCF bins are not shown. 

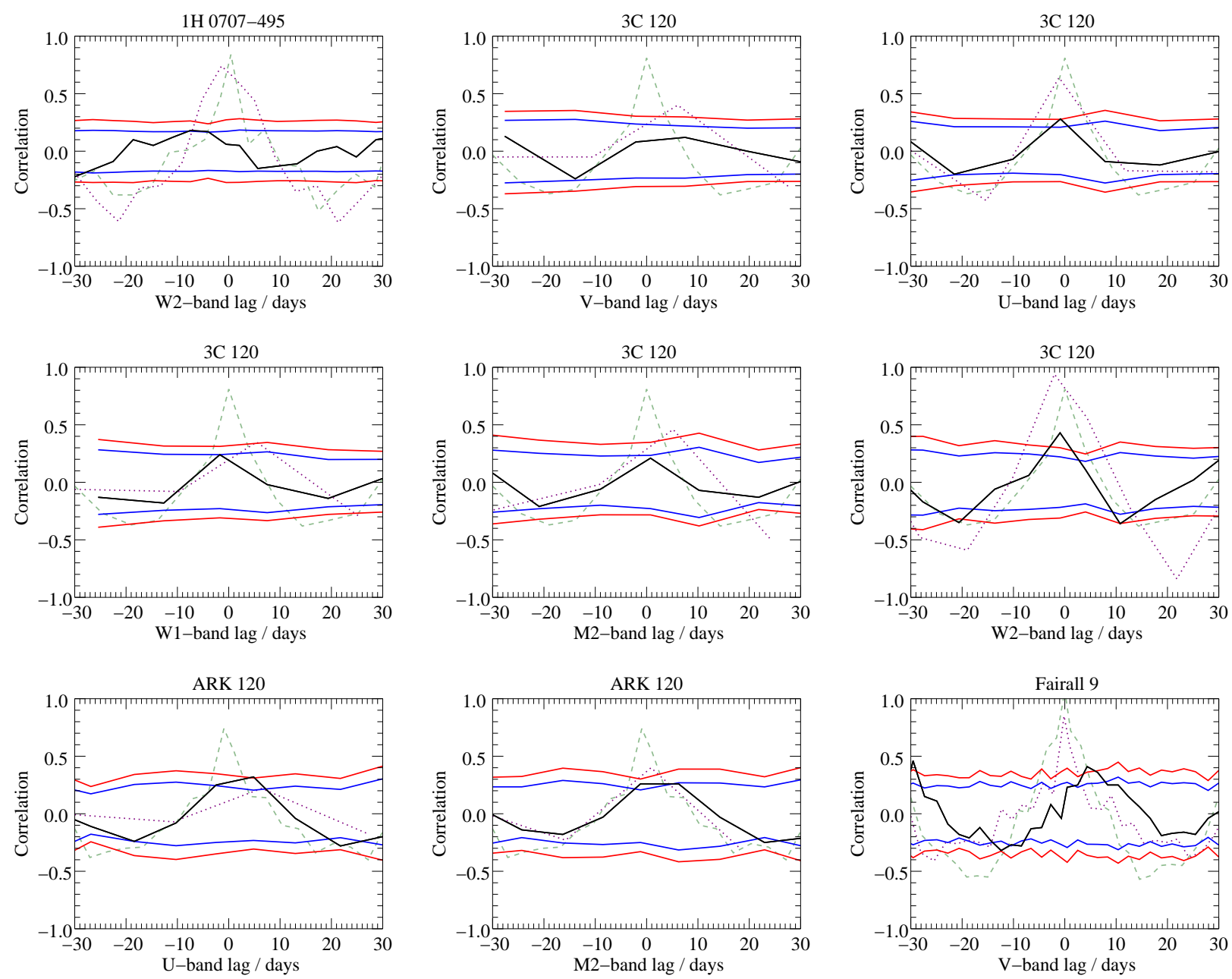

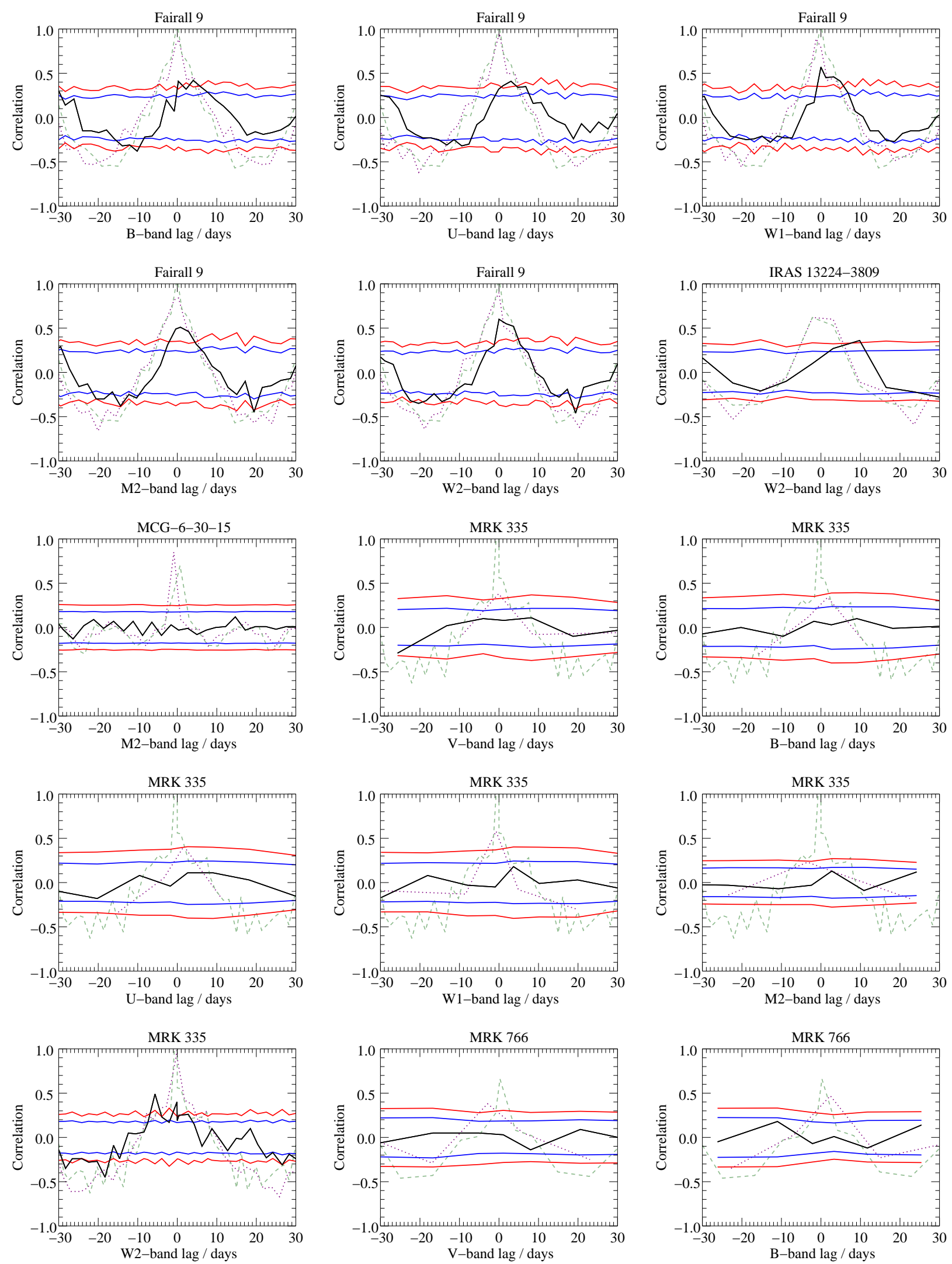

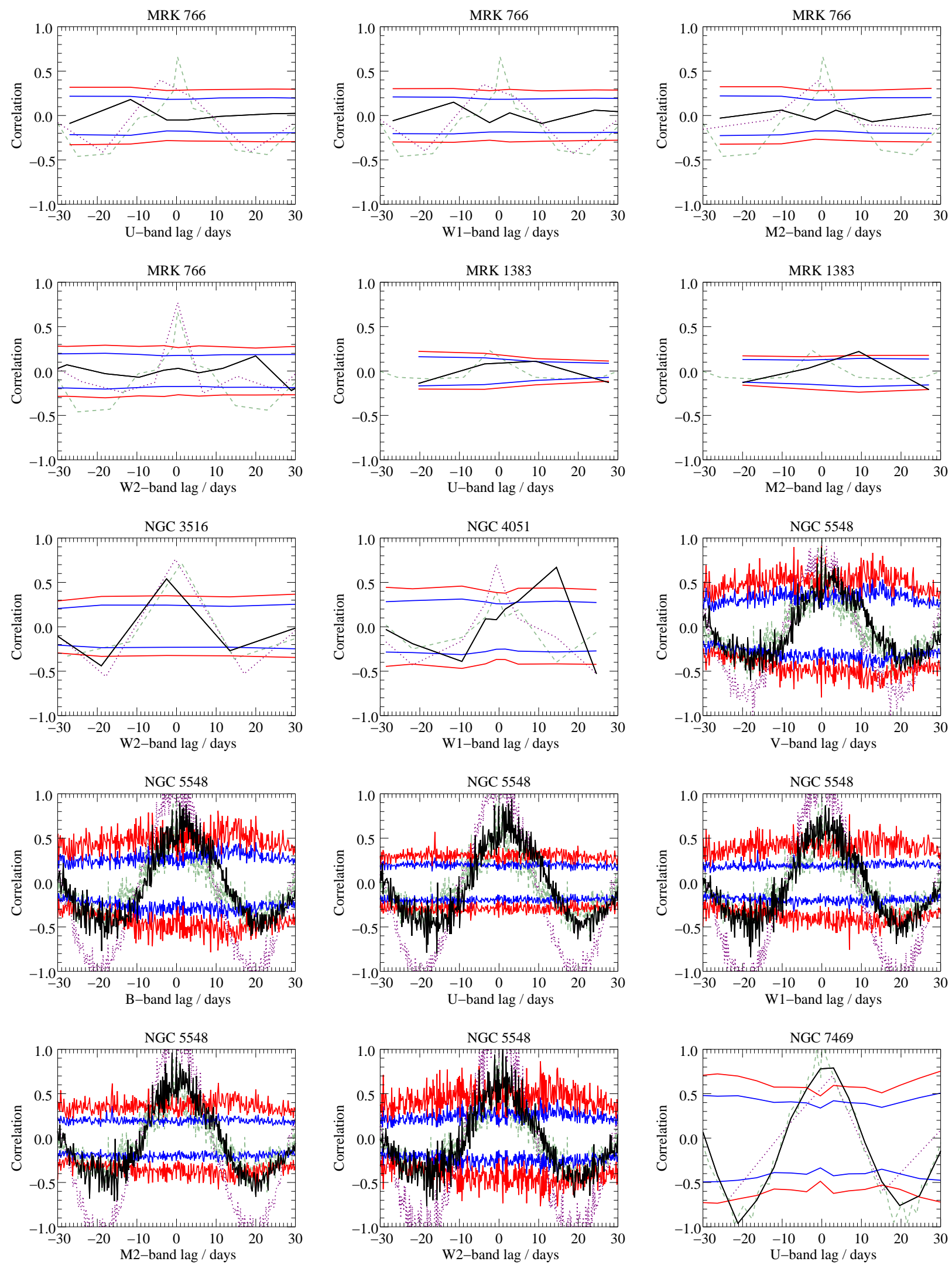

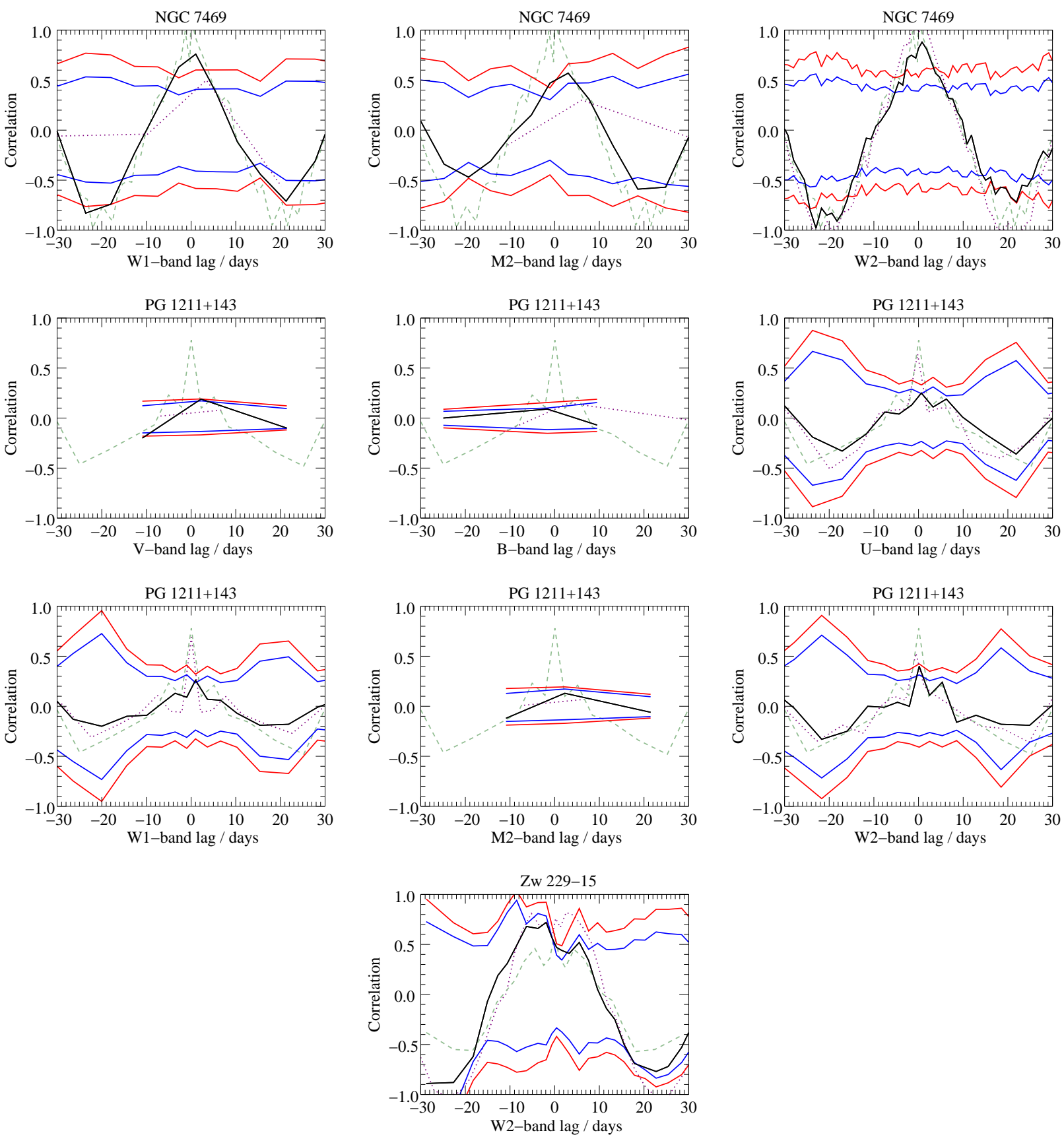


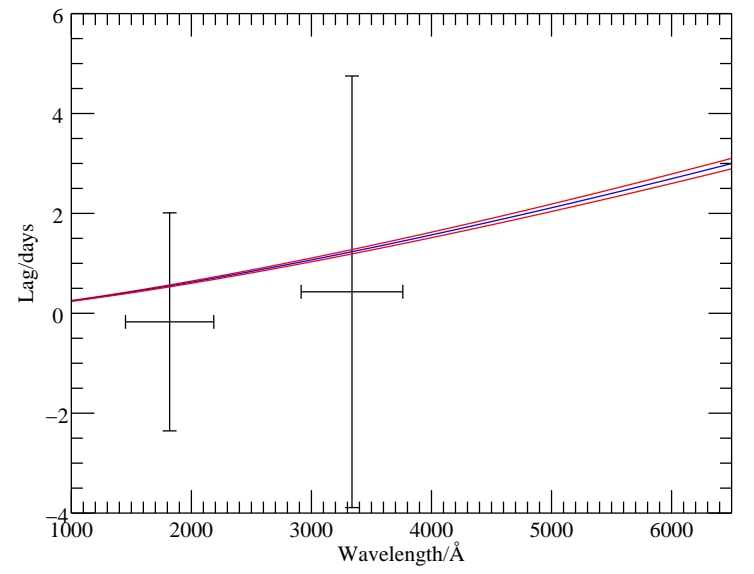

Figure D1. 3C 120

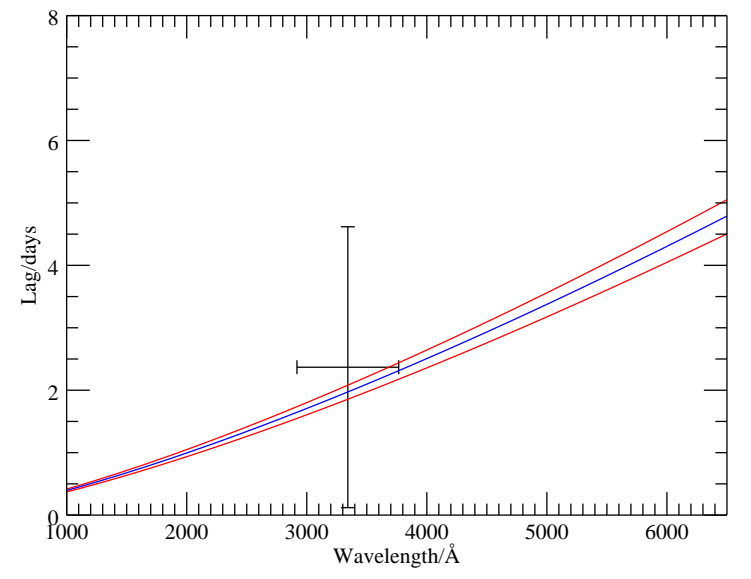

Figure D2. ARK 120

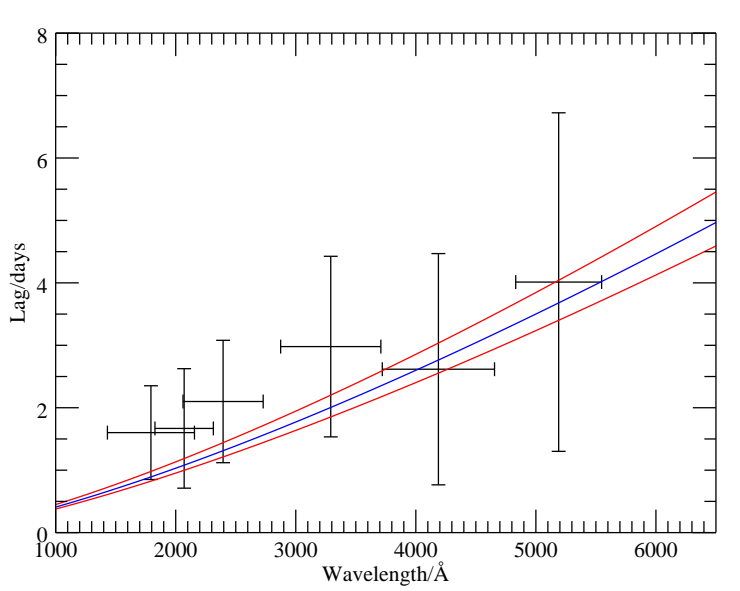

Figure D3. Fairall 9

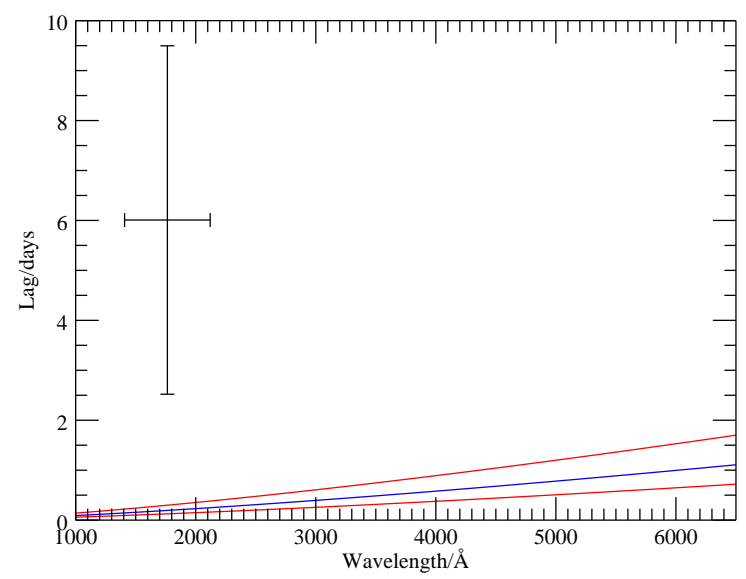

Figure D4. IRAS 13224-3809 


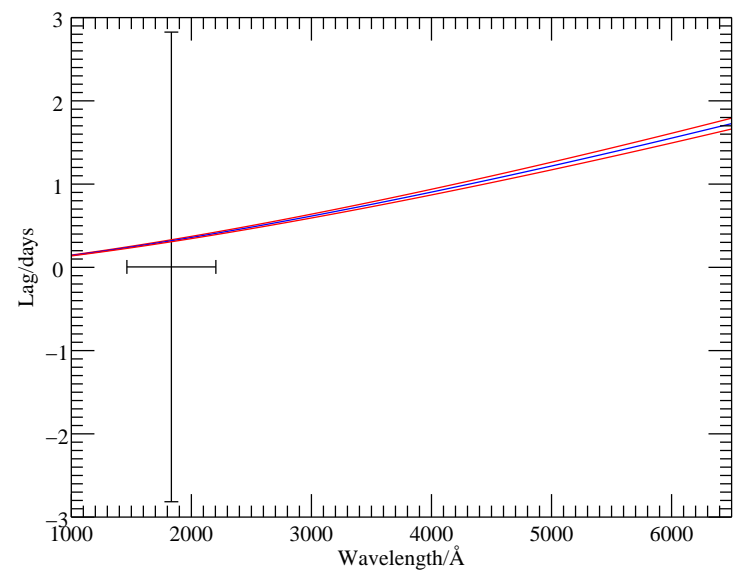

Figure D5. MRK 335

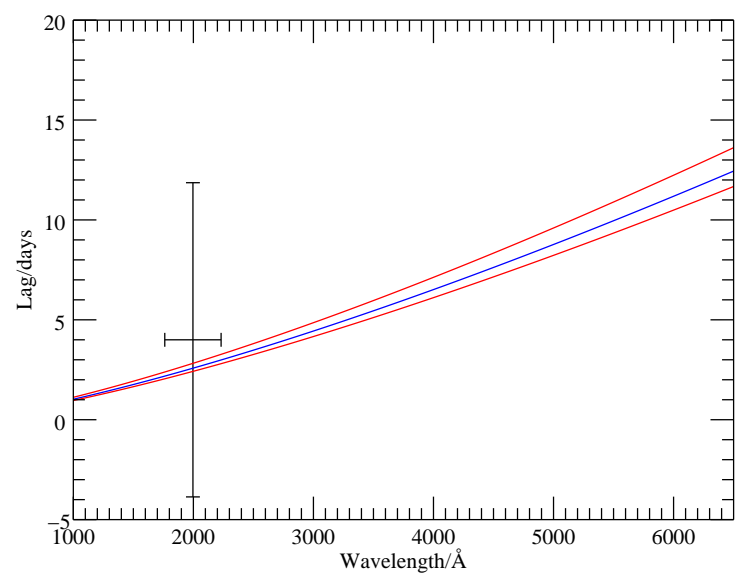

Figure D6. MRK 1383

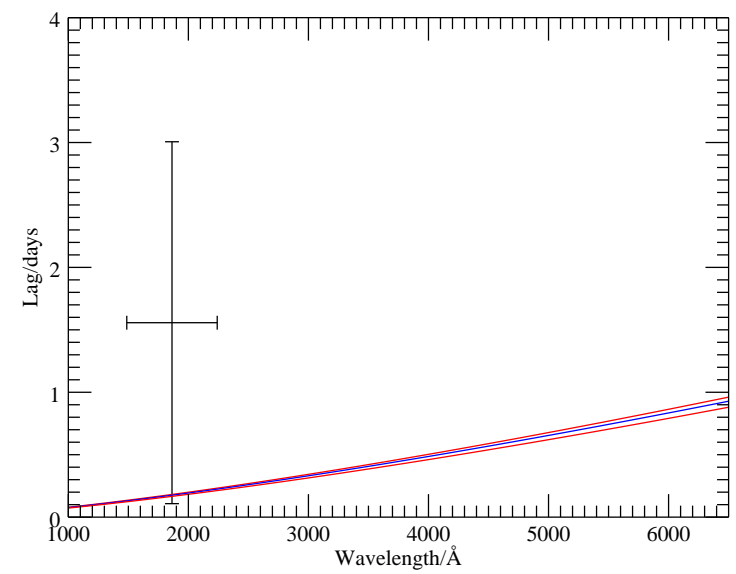

Figure D7. NGC 3516

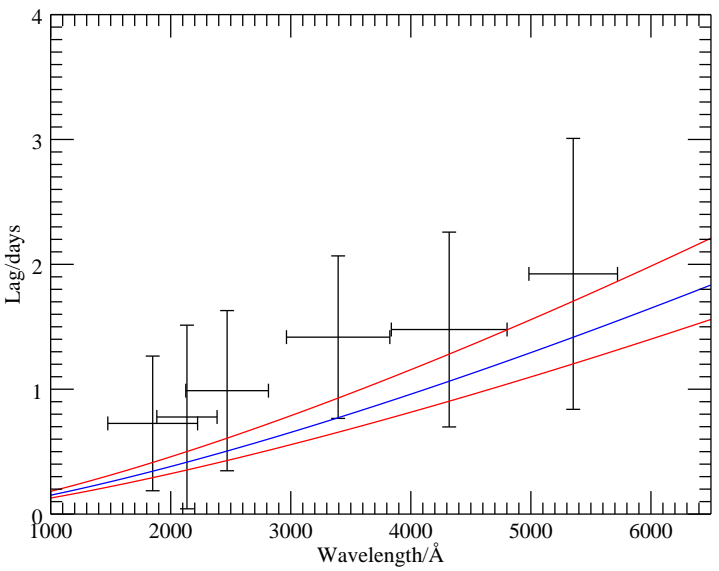

Figure D8. NGC 5548

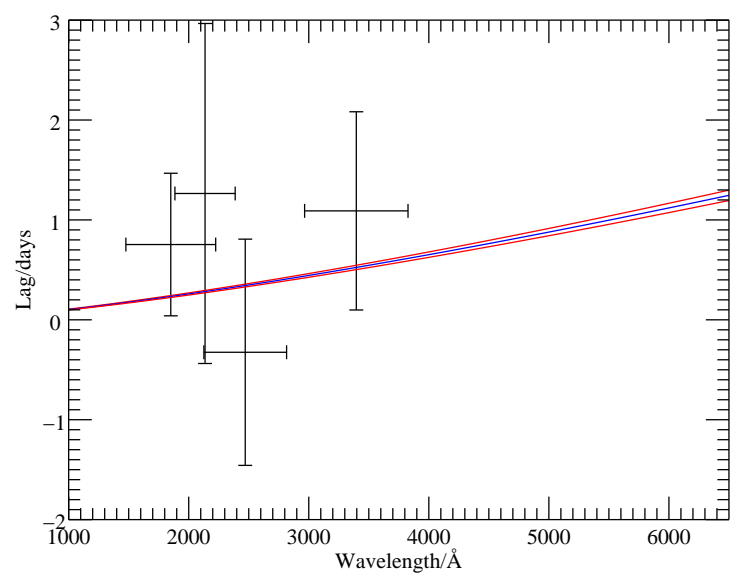

Figure D9. NGC 7469 